\title{
Scattering of water waves by rectangular thick barriers in presence of surface tension
}

\author{
Gour Das ${ }^{a}$, Rumpa Chakraborty ${ }^{b} 1$ \\ a. Department of Mathematics, Jadavpur University, Kolkata-700032, India. \\ b. Department of Mathematics, Diamond Harbour Women's University, South 24 \\ Parganas-743368, India
}

\begin{abstract}
The influence of surface tension over an oblique incident waves in presence of thick rectangular barriers present in water of uniform finite depth is discussed here. Three different structures of a bottom-standing submerged barrier, submerged rectangular block not extending down to the bottom and fully submerged block extending down to the bottom with a finite gap are considered. An appropriate multi-term Galekin approximation technique involving ultraspherical Gegenbauer polynomial is employed for solving the integral equations arising in the mathematical analysis. The reflection and transmission coefficients of the progressive waves for two-dimensional time harmonic motion are evaluated by utilizing linearized potential theory. The theoretical result is validated numerically and explained graphically in a number of figures. The present result will almost match analytically and graphically with those results already available in the literature without considering the effect of surface tension. From the graphical representation, it is clearly visible that the amplitude of reflection coefficient decreases with increasing values of surface tension. It is also seen that the presence of surface tension, the change of width, and the height of the thick barriers affect the nature of the reflection coefficients significantly.
\end{abstract}

Keywords: Oblique wave scattering, Surface tension, Thick rectangular barrier, Multiterm Galerkin approximation, Transmission and Reflection coefficients.

\section{Introduction}

Problems of water wave scattering by thin vertical barriers submerged in finite depth water are well studied in the literature using the linearized theory of water waves. For normal incident wave trains in finite depth water, the problems are solved explicitly employing various mathematical techniques by a number of researchers(cf. Packman and Williams [1], Losada et al. [2], Porter and Evans [3], Mandal and Dolai [4], Kanoria and Mandal [5], Banerjea et al. [6], Das et al. [7], Das, De and Mandal[8]). When the obstacle is in the form of a thick vertical barrier with a rectangular cross-section present in the water of uniform finite depth, the corresponding water wave scattering problems for normal incidence wave train have been investigated earlier by Mei and Black [9], Kanoria et al. [10]. Mei and Black [9] considered surface piercing and bottom standing thick vertical barriers and used variational formulations as the basis for numerical computations of the reflection and the transmission coefficients. Kanoria et al.[10] and Mandal and Kanoria [11] considered the problems of

\footnotetext{
${ }^{1}$ Corresponding author(e-mail: chak.rumpa@gmail.com tel: 9432852418)
} 
normal and oblique wave scattering by thick barriers respectively, wherein the barriers have four types of configurations such as surface-piercing or bottom standing thick barrier or a submerged block, or a thick wall with a gap. They used the multi-term Galerkin approximation method involving ultraspherical Gegenbauer polynomials for solving first kind integral equations arising in the mathematical analysis to obtain very accurate numerical estimates for the reflection coefficient. However, for oblique incidence of the wave trains in finite depth water, these problems cannot be solved explicitly.

None of the above research considered the effect of surface tension. Due to presence of cohesive forces in between water molecule in the upper surface of water, sometimes it's become necessary to consider the effect of surface tension on water wave scattering problems. A few of them such as Evans [12], [13], Rhodes-Robinson [14], [15], [16], Chakrabarti and Sahoo [17] included the effect of surface tension in water wave scattering problems. However, the scattering problem due to the thick rectangular barrier in presence of surface tension does not investigated yet. The amplitude and the frequency of the wave depend on both the surface tension and gravity. For this reason, it may not be possible to neglect the effect of surface tension while doing experimental study. As mentioned by Hocking and Mahdmina [18], another important reason for including surface tension is that in the absence of surface tension the transient motion initiated by an impulsive start is singular, but when the effect of surface tension is taken into account this singularity is removed. The uniqueness of the solution of the problem depends on the behavior of a special combination of the derivatives of the velocity potential at the edge because of the effect of surface tension as mentioned by Chakrabarti and Sahoo [17]. This is also an important reason for including the surface tension.

In our present study, we analyze the effect of surface tension on wave scattering problem by oblique incidence waves due to thick rectangular barrier. Three types of barrier, submerged bottom standing, submerged plate of finite height and fully submerged block with a gap are considered here. Due to geometrical symmetry about its central line, the scattering problems for all the cases are split into two separate boundary value problems in terms of symmetric and antisymmetric potential functions. Analytically the boundary value problems involving potential functions are reduced in to first kind integral equations using inverse Havelock inversion formulae. The integral equations are solved analytically by using multi term Galerkin approximation technique and there is used the ultra spherical Gegenbauer polynomial as a basis function as was done by Chakraborty and Mandal [19],[20], Newman [21] Sasmal and De [24], Paul and De[25]. The analytical result is explained numerically in terms of reflection coefficients of wave energy by a number of figures. The numerical results of the reflection coefficient for both types of thick barriers are obtained with almost six figure accuracies by taking only 6 terms in the multi-term Galerkin approximations and all of these results are depicted graphically against the non dimensional wave numbers. Some figures are compared with Kanoria et al.'s [10] result for normal incidence wave (incidence angle $\theta=0^{0}$ ) without taking the surface tension and a good agreement is achieved here. For large horizontal length of type I, II and III barriers, the numbers of zeros of the reflection coefficient as a function of wave number increases, which is also consistent with the observation of Kanoria et al.[10] for thick vertical barriers with free surface and Newman [21] for 
long bottom obstacles. In the present study, the energy balance relation involving reflection and transmission coefficients is also satisfied numerically. These all results provide the checks of the correctness of the present numerical method utilized here. The new outcome of this article is that the surface tension together with the change of width of the barrier affects the amplitude of reflection coefficients significantly.

\section{Problem Construction}

We consider a time harmonic wave motion of angular frequency $\sigma$ under the action of both gravity with acceleration $g$ and surface tension $T$ in region of finite water depth $h$ in which a thick rectangular barrier of uniform width $2 b$ is immersed. Here water is taken as an inviscid, homogeneous fluid with irrotational motion.

A rectangular cartesian coordinate system is chosen, where $y$ axis is taken vertically downwards and the $(x, z)$ plane corresponds to the undisturbed surface. Here we study three different cases of barrier placement(as shown in fig 1), the barrier may occupy the region $-b \leq x \leq b$ with (I) $y \in L=L_{1}(=(c, h))$, (II) $y \in L=L_{2}(=(a, c)$ ), with $0 \leq a \leq c \leq h$ and (III) $y \in L=L_{3}(=(a, c)+(d, h))$, with $0 \leq a \leq c \leq d \leq h$. In the present computation $L_{1}, L_{2}$ and $L_{3}$, corresponding type-I, type II and type-III barrier configuration respectively.

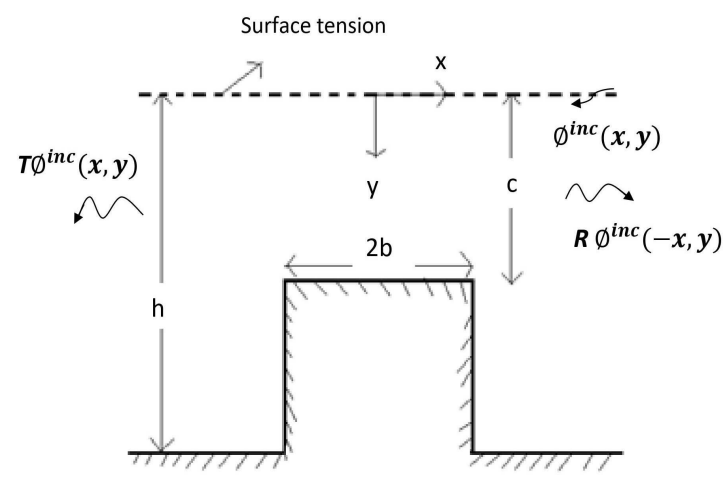

Type-I

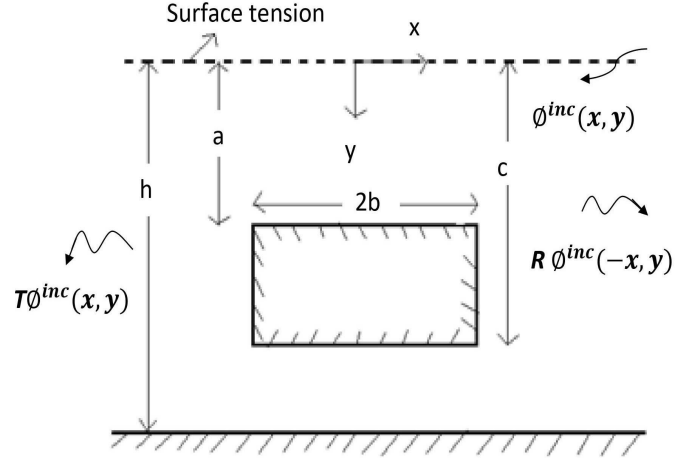

Type-II

Here we consider a harmonically time dependent progressive wave, obliquely incident from negative infinity and its direction makes an angle $\theta$ with the horizontal $x$ - axis. We denote the wave number by $\gamma_{0}$, where $\gamma_{0}=\frac{2 \pi}{\lambda}, \lambda$ is the wave length of incident wave. The incident wave number $\gamma_{0}$ satisfy the transcendental equation

$$
\gamma\left(1+M \gamma^{2}\right) \tanh \gamma h=K, K=\frac{\sigma^{2}}{g},
$$

$M=\frac{\tau}{\rho g}, \tau$ is the coefficient of surface tension at the free surface of the ocean, $\rho$ is the density of the fluid.

The position of roots of the transcendental equation (1) is shown numerically in Fig. 2 using a contour plot. To draw the contour, we consider some fixed values of $\frac{M}{h^{2}}(=2)$ and 


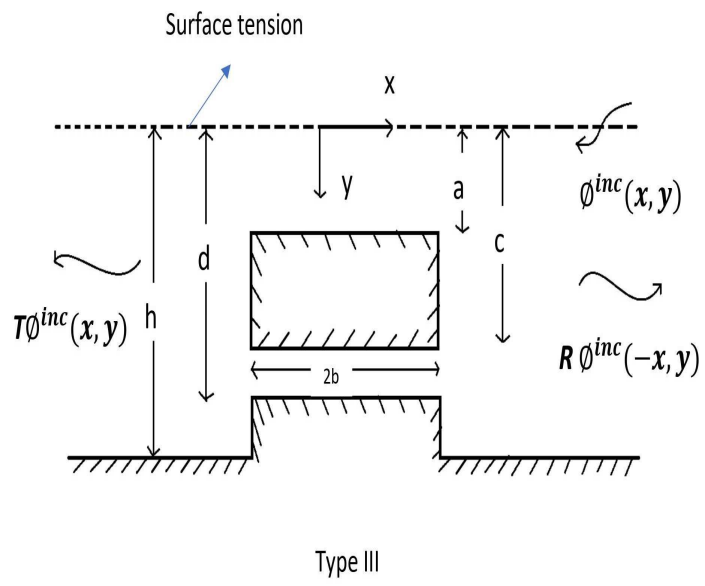

Figure 1: Diagram of thick barriers

$K h=0.8$. In the figure, the black dots signify the position of the roots and it is very clear that, in presence of surface tension, the dispersion equation has two real roots $\pm \gamma_{0}$ and infinitely many purely imaginary roots $\pm i \gamma_{n}, n=1,2, \ldots\left(\gamma_{n}>0\right)$. [cf. Chakrabarti and Sahoo[17], Sasmal, Paul and De[22], Paul et. al.,[23]]. Assuming linear theory, a train of incident wave is represented by $\Re\left\{\phi^{i n c}(x, y) e^{i(\nu z-\sigma t)}\right\}$ where

$$
\phi^{i n c}(x, y)=\frac{2 \cosh \gamma_{0}(h-y) e^{-i \mu(x-b)}}{\cosh \gamma_{0} h}
$$

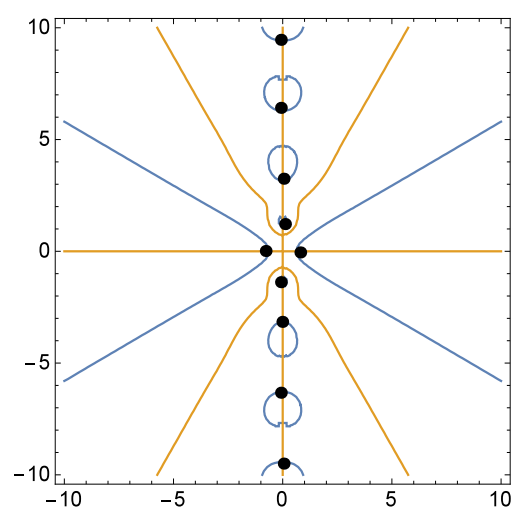

Figure 2: Contour plot $\Gamma$ of the roots of the dispersion equation for $\frac{M}{h^{2}}=2, K h=0.8$.

Here $\mu=\gamma_{0} \cos \theta$ and $\nu=\gamma_{0} \sin \theta$ where $0<\theta<\pi / 2$ and $e^{i(\nu z)}$ is the $\mathrm{z}$ dependence term of the fluid. Due to geometrical symmetry of the problem, the $z$ dependent term can be eliminated by assuming the velocity potential to be of the form $\Re\left\{\phi^{i n c}(x, y) e^{i(\nu z-\sigma t)}\right\}$. Henceforth, the factor $e^{i(\nu z-\sigma t)}$ will be omitted.

Then velocity potential function $\phi(x, y)$ satisfies the boundary value problem

$$
\left(\nabla^{2}-\nu^{2}\right) \phi=0 \quad \text { in the fluid region }
$$




$$
\begin{gathered}
K \phi+\frac{\partial \phi}{\partial y}+M \frac{\partial^{3} \phi}{\partial y^{3}}=0 \quad \text { on } \quad y=0, \quad|x|<\infty \\
\phi_{x}=0 \text { on } \quad x= \pm b, \quad y \in L_{j}, j=1,2,3 \\
r^{\frac{1}{3}} \nabla \phi \text { is bounded as } r \rightarrow 0,
\end{gathered}
$$

$r$ is the distance from a submerged edge of the thick barrier,

$$
\begin{gathered}
\phi_{y}=0 \quad \text { on } y=l_{j}, \quad|x|<b, \quad \text { for } \mathrm{j} \text {-th type of barrier, } j=1,2,3 \\
\phi_{y}=0 \quad \text { on } y=h, \quad\left\{\begin{array}{l}
|x|>b \text { for type I and III barrier } \\
|x|<\infty \text { for type II barrier }
\end{array}\right.
\end{gathered}
$$

and finally

$$
\phi(x, y) \sim\left\{\begin{array}{l}
\phi^{i n c}(x, y)+R \phi^{i n c}(-x, y) \text { as } x \rightarrow \infty \\
T \phi^{i n c}(x, y) \quad \text { as } x \rightarrow-\infty
\end{array}\right.
$$

where $R$ and $T$ is the unknown reflection and transmission coefficients respectively and is to be determined for each barrier configuration. In (7), $l_{1}=c ; l_{2}=a, c ; l_{3}=a, c, d$; corresponding to type I, II, III barriers configurations respectively depicted in Figure 1.

\section{Method of solution}

Presence of the uniform geometrical structure of the rectangular barrier about $x=0 ; \phi(x, y)$ can be divided into symmetric and antisymmetric parts, $\phi^{S M M}(x, y)$ and $\phi^{A N S M M}(x, y)$, respectively, so that

$$
\phi(x, y)=\phi^{S M M}(x, y)+\phi^{A N S M M}(x, y)
$$

where

$$
\phi^{S M M}(-x, y)=\phi^{S M M}(x, y), \phi^{A N S M M}(-x, y)=-\phi^{A N S M M}(x, y)
$$

Therefore, we consider only the region $x \geq 0$. Now $\phi^{\operatorname{SMM}, A N S M M}(x, y)$ satisfy (3) to (8) together with

$$
\phi_{x}^{S M M}(0, y)=0 \text { and } \phi^{A N S M M}(0, y)=0, \quad 0<y<h .
$$

Let the behavior of $\phi^{S M M, A N S M M}(x, y)$ for large $x$ be represented by

$$
\phi^{S M M, A N S M M}(x, y) \sim \frac{\cosh \gamma_{0}(h-y)}{\cosh \gamma_{0} h}\left\{e^{-i \mu(x-b)}+R^{S M M, A N S M M} e^{i \mu(x-b)}\right\} \quad \text { as } \quad x \rightarrow \infty
$$

where $R^{S M M}$ and $R^{A N S M M}$ are unknown constants. These constants are related to $R$ and $T$ by

$$
R, T=\frac{1}{2}\left(R^{S M M} \pm R^{A N S M M}\right) e^{-2 i \mu b}
$$

Now the eigen function expansions of $\phi^{S M M, A N S M M}(x, y)$ satisfying (3) to (8) and (12) for $x>0$ in the different regions for each barrier configuration are below. 
Region I $(x>b, 0<y<h)$ :

$$
\begin{gathered}
\phi^{S M M, A N S M M}(x, y)=\frac{\cosh \gamma_{0}(h-y)}{\cosh \gamma_{0} h}\left\{e^{-i \mu(x-b)}+R^{S M M, A N S M M} e^{i \mu(x-b)}\right\} \\
+\sum_{n=1}^{\infty} \mathcal{A}_{n}^{S M M, A N S M M} \cos \gamma_{n}(h-y) e^{-s_{n}(x-b)}
\end{gathered}
$$

where $\gamma_{n}(n=1,2, \ldots)$ are the real positive roots of the equation

$$
x\left(1-M x^{2}\right) \tan x h+K=0 .
$$

and $s_{n}=\left(\gamma_{n}^{2}+\nu^{2}\right)^{\frac{1}{2}}$.

$$
\text { Region II }\left(0<x<b, \quad y \in(0, h)-L_{j}, \quad j=1,2,3\right) \text { : }
$$

\section{Type I:}

For $y \in(0, c), \phi^{S M M}(x, y)$ and $\phi^{A N S M M}(x, y)$ are given by

$$
\begin{gathered}
\left(\begin{array}{l}
\phi^{S M M}(x, y) \\
\phi^{A N S M M}(x, y)
\end{array}\right)=\frac{\cosh \alpha_{0}(c-y)}{\cosh \alpha_{0} c}\left(\begin{array}{l}
\mathcal{B}_{0}^{S M M} \cos \left(\alpha_{0}^{2}-\nu^{2}\right)^{\frac{1}{2}} x \\
\mathcal{B}_{0}^{A N S M M} \sin \left(\alpha_{0}^{2}-\nu^{2}\right)^{\frac{1}{2}} x
\end{array}\right) \\
+\sum_{n=1}^{\infty}\left(\begin{array}{l}
\mathcal{B}_{n}^{S M M} \cosh t_{n} x \\
\mathcal{B}_{n}^{A N S M M} \sinh t_{n} x
\end{array}\right) \cos \alpha_{n}(c-y)
\end{gathered}
$$

where $\pm \alpha_{0}, \pm i \alpha_{n}(n=1,2, \ldots)$ are the roots of the equation

$$
\alpha\left(1+M \alpha^{2}\right) \tanh \alpha c=K .
$$

and

$$
t_{n}=\left(\alpha_{n}^{2}+\nu^{2}\right)^{\frac{1}{2}},(n=1,2, . .) .
$$

\section{Type II:}

For $y \in(0, a)+(c, h), \phi^{S M M, A N S M M}(x, y)$ will have two types of expressions depending on whether $0<y<a$ or $c<y<h$. For $0<y<a$ the expressions of $\phi^{S M M, A N S M M}(x, y)$ are similar to equation (17) with $\mathcal{B}_{n}^{S M M, A N S M M}$ replaced by $D_{n}^{S M M, A N S M M}, \alpha_{n}$ replaced by $\beta_{n}, t_{n}$ replaced by $u_{n}$ and $c$ replaced by $a$, where $\pm \beta_{0}, \pm i \beta_{n}(n=1,2, \ldots)$ are the roots of the equation

$$
\begin{gathered}
\quad \beta\left(1+M \beta^{2}\right) \tanh \beta a=K \\
\text { and } \quad u_{n}=\left(\beta_{n}^{2}+\nu^{2}\right)^{\frac{1}{2}},(n=1,2, . .) .
\end{gathered}
$$

For $c<y<h$ the expressions of $\phi^{S M M, A N S M M}(x, y)$ are given by

$$
\left(\begin{array}{l}
\phi^{S M M}(x, y) \\
\phi^{A N S M M}(x, y)
\end{array}\right)=\left(\begin{array}{l}
0 \\
E_{0}^{A N S M M} x
\end{array}\right)+\sum_{n=1}^{\infty}\left(\begin{array}{l}
E_{n}^{S M M} \cosh \frac{n \pi x}{h-c} \\
E_{n}^{A N S M M} \sinh \frac{n \pi x}{h-c}
\end{array}\right) \cos \frac{n \pi(y-c)}{h-c}
$$

\section{Type III:}


For $y \in(0, a)+(c, d), \phi^{S M M, A N S M M}(x, y)$ will have two types of expressions depending on whether $0<y<a$ or $c<y<d$. For $0<y<a$ the expressions of $\phi^{S M M, A N S M M}(x, y)$ are similar to equation (17) with $\mathcal{B}_{n}^{S M M, A N S M M}$ replaced by $D_{n}^{S M M, A N S M M}, \alpha_{n}$ replaced by $\beta_{n}, t_{n}$ replaced by $u_{n}$ and c replaced by a, where $\pm \beta_{0}, \pm i \beta_{n}(n=1,2, \ldots)$ are the roots of the equation (20) and $u_{n}$ are given by (21).

For $c<y<d$ the expressions of $\phi^{S M M, A N S M M}(x, y)$ are given by

$$
\left(\begin{array}{l}
\phi^{S M M}(x, y) \\
\phi^{A N S M M}(x, y)
\end{array}\right)=\left(\begin{array}{l}
U_{0}^{S M M} \cosh \nu x \\
U_{0}^{A N S M M} \sinh \nu x
\end{array}\right)+\sum_{n=1}^{\infty}\left(\begin{array}{l}
U_{n}^{S M M} \cosh \zeta_{n} x \\
U_{n}^{A N S M M} \sinh \zeta_{n} x
\end{array}\right) \cos \frac{n \pi(d-y)}{d-c}
$$

where

$$
\zeta_{n}=\sqrt{\left(\frac{n \pi}{d-c}\right)^{2}+\nu^{2}}
$$

Now let us define

$$
\phi_{x}^{S M M, A N S M M}(b+0, y)=f^{S M M, A N S M M}(y), 0<y<h,
$$

Then

$$
f^{S M M, A N S M M}(y)=0, \quad \text { for } y \in L_{j}
$$

and $\quad \phi_{x}^{S M M, A N S M M}(b-0, y)=f^{S M M, A N S M M}(y)$, for $y \in(0, h)-L_{j}, j=1,2,3$.

Also, due to the edge condition described in equation (6), we must have the requirement that

$$
f^{S M M, A N S M M}(y)=O\left(\left|y-l_{j}\right|^{-\frac{1}{3}}\right) \quad \text { as } y \rightarrow l_{j}, j=1,2,3 .
$$

Using the expression of equation (15) for $\phi^{S M M, A N S M M}(y)$ in (25) and followed by Havelock inversion formula gives

$$
1-R^{S M M, A N S M M}=\frac{4 i \gamma_{0}}{\delta_{0} \mu} \cosh \gamma_{0} h \int_{a}^{h} f^{S M M, A N S M M}(y) \cosh \gamma_{0}(h-y) d y
$$

and

$$
\mathcal{A}_{n}^{S M M, A N S M M}=-\frac{4 \gamma_{n}}{\delta_{n} s_{n}} \int_{a}^{h} f^{S M M, A N S M M}(y) \cos \gamma_{n}(h-y) d y
$$

with $\delta_{0}=2 \gamma_{0} h+\sinh 2 \gamma_{0} h ; \delta_{n}=2 \gamma_{n} h+\sin 2 \gamma_{n} h \quad(n=1,2, \ldots)$.

\subsection{Evaluate the values of constants for region II}

\section{For Type I}

Substituting the equation (17) in condition (27) and using Fourier cosine inversion, we get

$$
\mathcal{B}_{0}^{S M M, A N S M M}=\frac{4 \alpha_{0} \cosh \alpha_{0} c}{\lambda_{0}\left(\alpha_{0}^{2}-\nu^{2}\right)^{\frac{1}{2}}}\left(\frac{1}{-\sin \left(\alpha_{0}^{2}-\nu^{2}\right)^{\frac{1}{2}} b}, \frac{1}{\cos \left(\alpha_{0}^{2}-\nu^{2}\right)^{\frac{1}{2}} b}\right)
$$




$$
\begin{gathered}
\times \int_{0}^{c} f^{S M M, A N S M M}(y) \cosh \alpha_{0}(c-y) d y \\
\mathcal{B}_{n}^{S M M, A N S M M}=\frac{4 \alpha_{n}}{\lambda_{n} t_{n}}\left(\frac{1}{\sinh t_{n} b}, \frac{1}{\cosh t_{n} b}\right) \int_{0}^{c} f^{S M M, A N S M M}(y) \cos \alpha_{n}(c-y) d y
\end{gathered}
$$

with $\lambda_{0}=2 \alpha_{0} c+\sinh \alpha_{0} c ; \lambda_{n}=2 \alpha_{n} c+\sin 2 \alpha_{n} c \quad(n=1,2, \ldots)$.

\section{For Type II}

Here we can derive the constants $D_{n}^{S M M, A N S M M}$ from $\mathcal{B}_{n}^{S M M, A N S M M}$ by replacing $\alpha_{n}$ by $\beta_{n}$, $t_{n}$ by $u_{n}, \lambda_{n}$ by $\epsilon_{n}$ and $c$ by $a$ with $\epsilon_{0}=2 \beta_{0} a+\sinh \beta_{0} a ; \epsilon_{n}=2 \beta_{n} a+\sin 2 \beta_{n} a \quad(n=1,2, \ldots)$.

$$
\begin{gathered}
\text { and } E_{0}^{A N S M M}=\frac{1}{h-c} \int_{c}^{h} f^{A N S M M}(y) d y \\
E_{n}^{S M M, A N S M M}=\frac{2}{n \pi}\left(\frac{1}{\sinh \frac{n \pi b}{h-c}}, \frac{1}{\cosh \frac{n \pi b}{h-c}}\right) \int_{c}^{h} f^{S M M, A N S M M}(y) \cos \frac{n \pi(y-c)}{h-c} d y
\end{gathered}
$$

and $f^{S M M}(y)$ for type II barrier must satisfy the condition

$$
\int_{c}^{h} f^{S M M}(y) d y=0
$$

\section{For Type III}

Here the constants $D_{n}^{S M M, A N S M M}$ are obtained from $\mathcal{B}_{n}^{S M M, A N S M M}$ by changing $\alpha_{n}$ by $\beta_{n}$, $t_{n}$ by $u_{n}, \lambda_{n}$ by $\epsilon_{n}$ and $c$ by $a$ with $\epsilon_{0}=2 \beta_{0} a+\sinh \beta_{0} a ; \epsilon_{n}=2 \beta_{n} a+\sin 2 \beta_{n} a \quad(n=1,2, \ldots)$. and

$$
\begin{gathered}
U_{0}^{S M M, A N S M M}=\frac{1}{\nu(d-c)}\left(\frac{1}{\sinh \nu b}, \frac{1}{\cosh \nu b}\right) \int_{c}^{d} f^{S M M, A N S M M}(y) d y \\
U_{n}^{S M M, A N S M M}=\frac{2}{\zeta_{n}(d-c)}\left(\frac{1}{\sinh \zeta_{n} b}, \frac{1}{\cosh \zeta_{n} b}\right) \int_{c}^{d} f^{S M M, A N S M M}(y) \cos \frac{n \pi(d-y)}{d-c} d y
\end{gathered}
$$

\subsection{Formation of integral equation:}

Now equating $\phi^{S M M, A N S M M}(x, y)$ through $x=b$, we get

$$
\phi_{x}^{S M M, A N S M M}(b+0, y)=\phi_{x}^{S M M, A N S M M}(b-0, y),
$$

for $y \in(0, h)-L_{j}$, and it ultimately constructs the integral equation

$$
\int_{(0, h)-L_{j}} F^{S M M, A N S M M}(u) N^{S M M, A N S M M}(y, u) d u=\frac{\cosh \gamma_{0}(h-y)}{\cosh \gamma_{0} h}, \text { for } y \in(0, h)-L_{j}
$$




$$
\text { where } F^{S M M, A N S M M}(y)=\frac{4 \gamma_{0} \cosh ^{2} \gamma_{0} h}{\delta_{0} \mu\left(1+R^{S M M, A N S M M}\right)} f^{S M M, A N S M M}(y),
$$

and $N^{S M M, A N S M M}(y, u)\left(y, u \in(0, h)-L_{j}\right)(j=1,2,3)$ are real symmetric in $y$ and $u$ and their constructions are given below

\section{Type I}

$$
\begin{aligned}
& N^{S M M}(y, u)=\frac{\delta_{0} \mu}{\gamma_{0} \cosh ^{2} \gamma_{0} h}\left[\sum _ { n = 1 } ^ { \infty } \left\{\frac{\gamma_{n} \cos \gamma_{n}(h-y) \cos \gamma_{n}(h-u)}{\delta_{n} s_{n}}\right.\right. \\
& \left.+\frac{\alpha_{n} \operatorname{coth} t_{n} b \cos \alpha_{n}(c-y) \cos \alpha_{n}(c-u)}{\lambda_{n} t_{n}}\right\} \\
& \left.+\left(-\cot \left(\alpha_{0}^{2}-\nu^{2}\right)^{\frac{1}{2}} b\right) \frac{\alpha_{0} \cosh \alpha_{0}(c-y) \cosh \alpha_{0}(c-u)}{\lambda_{0}\left(\alpha_{0}^{2}-\nu^{2}\right)^{\frac{1}{2}}}\right], 0<y, u<h . \\
& N^{A N S M M}(y, u)=\frac{\delta_{0} \mu}{\gamma_{0} \cosh ^{2} \gamma_{0} h}\left[\sum _ { n = 1 } ^ { \infty } \left\{\frac{\gamma_{n} \cos \gamma_{n}(h-y) \cos \gamma_{n}(h-u)}{\delta_{n} s_{n}}\right.\right. \\
& \left.+\frac{\alpha_{n} \tanh t_{n} b \cos \alpha_{n}(c-y) \cos \alpha_{n}(c-u)}{\lambda_{n} t_{n}}\right\} \\
& \left.+\left(\tan \left(\alpha_{0}^{2}-\nu^{2}\right)^{\frac{1}{2}} b\right) \frac{\alpha_{0} \cosh \alpha_{0}(c-y) \cosh \alpha_{0}(c-u)}{\lambda_{0}\left(\alpha_{0}^{2}-\nu^{2}\right)^{\frac{1}{2}}}\right], 0<y, u<h .
\end{aligned}
$$

\section{Type II}

For $y, u \in(0, a)+(c, h)$, we get three different cases for $N^{S M M, A N S M M}(y, u)$

Case I:

$y, u \in(0, a)$

$$
\begin{gathered}
N^{S M M}(y, u)=\frac{\delta_{0} \mu}{\gamma_{0} \cosh ^{2} \gamma_{0} h}\left[\sum _ { n = 1 } ^ { \infty } \left\{\frac{\gamma_{n} \cos \gamma_{n}(h-y) \cos \gamma_{n}(h-u)}{\delta_{n} s_{n}}\right.\right. \\
\left.+\frac{\beta_{n} \operatorname{coth} u_{n} b \cos \beta_{n}(a-y) \cos \beta_{n}(a-u)}{\epsilon_{n} u_{n}}\right\} \\
\left.+\left(-\cot \left(\beta_{0}^{2}-\nu^{2}\right)^{\frac{1}{2}} b\right) \frac{\beta_{0} \cosh \beta_{0}(a-y) \cosh \beta_{0}(a-u)}{\epsilon_{0}\left(\beta_{0}^{2}-\nu^{2}\right)^{\frac{1}{2}}}\right], 0<y, u<h . \\
N^{A N S M M}(y, u)=\frac{\delta_{0} \mu}{\gamma_{0} \cosh ^{2} \gamma_{0} h}\left[\sum _ { n = 1 } ^ { \infty } \left\{\frac{\gamma_{n} \cos \gamma_{n}(h-y) \cos \gamma_{n}(h-u)}{\delta_{n} s_{n}}\right.\right. \\
\left.+\frac{\beta_{n} \tanh u_{n} b \cos \beta_{n}(a-y) \cos \beta_{n}(a-u)}{\epsilon_{n} u_{n}}\right\}
\end{gathered}
$$




$$
\left.+\left(\tan \left(\beta_{0}^{2}-\nu^{2}\right)^{\frac{1}{2}} b\right) \frac{\beta_{0} \cosh \beta_{0}(a-y) \cosh \beta_{0}(a-u)}{\epsilon_{0}\left(\beta_{0}^{2}-\nu^{2}\right)^{\frac{1}{2}}}\right], 0<y, u<h .
$$

\section{Case II}

$y, u \in(c, h)$

$$
\begin{aligned}
N^{S M M}(y, u) & =\frac{\delta_{0} \mu}{\gamma_{0} \cosh ^{2} \gamma_{0} h}\left[\sum _ { n = 1 } ^ { \infty } \left(\frac{\gamma_{n} \cos \gamma_{n}(h-y) \cos \gamma_{n}(h-u)}{\delta_{n} s_{n}}+\right.\right. \\
& \left.\left.\frac{1}{2 n \pi} \operatorname{coth} \frac{n \pi b}{h-c} \cos \frac{n \pi(y-c)}{h-c} \cos \frac{n \pi(u-c)}{h-c}\right)\right] \\
N^{A N S M M}(y, u)= & \frac{\delta_{0} \mu}{\gamma_{0} \cosh ^{2} \gamma_{0} h}\left[\frac{b}{4(h-c)}+\sum_{n=1}^{\infty}\left(\frac{\gamma_{n} \cos \gamma_{n}(h-y) \cos \gamma_{n}(h-u)}{\delta_{n} s_{n}}+\right.\right. \\
& \left.\left.\frac{1}{2 n \pi} \tanh \frac{n \pi b}{h-c} \cos \frac{n \pi(y-c)}{h-c} \cos \frac{n \pi(u-c)}{h-c}\right)\right]
\end{aligned}
$$

\section{Case III}

$y \in(0, a), u \in(c, h)$ or $y \in(c, h), u \in(0, a)$

$$
N^{S M M, A N S M M}(y, u)=\frac{\delta_{0} \mu}{\gamma_{0} \cosh ^{2} \gamma_{0} h}\left[\sum_{n=1}^{\infty} \frac{\gamma_{n} \cos \gamma_{n}(h-y) \cos \gamma_{n}(h-u)}{\delta_{n} s_{n}}\right]
$$

\section{Type III}

For $y, u \in(0, a)+(c, d)$, we have three different cases for $N^{S M M, A N S M M}(y, u)$

Case I:

$y, u \in(0, a)$

$$
\begin{gathered}
N^{S M M}(y, u)=\frac{\delta_{0} \mu}{\gamma_{0} \cosh ^{2} \gamma_{0} h}\left[\sum _ { n = 1 } ^ { \infty } \left\{\frac{\gamma_{n} \cos \gamma_{n}(h-y) \cos \gamma_{n}(h-u)}{\delta_{n} s_{n}}\right.\right. \\
\left.+\frac{\beta_{n} \operatorname{coth} u_{n} b \cos \beta_{n}(a-y) \cos \beta_{n}(a-u)}{\epsilon_{n} u_{n}}\right\} \\
+\left(-\cot \left(\beta_{0}^{2}-\nu^{2}\right)^{\frac{1}{2}} b\right) \frac{\beta_{0} \cosh \beta_{0}(a-y) \cosh \beta_{0}(a-u)}{\left.\epsilon_{0}\left(\beta_{0}^{2}-\nu^{2}\right)^{\frac{1}{2}}\right], 0<y, u<h .} \\
N^{A N S M M}(y, u)=\frac{\delta_{0} \mu}{\gamma_{0} \cosh \gamma_{0} h}\left[\sum _ { n = 1 } ^ { \infty } \left\{\frac{\gamma_{n} \cos \gamma_{n}(h-y) \cos \gamma_{n}(h-u)}{\delta_{n} s_{n}}\right.\right. \\
\left.+\frac{\beta_{n} \tanh u_{n} b \cos \beta_{n}(a-y) \cos \beta_{n}(a-u)}{\epsilon_{n} u_{n}}\right\} \\
\left.+\left(\tan \left(\beta_{0}^{2}-\nu^{2}\right)^{\frac{1}{2}} b\right) \frac{\beta_{0} \cosh \beta_{0}(a-y) \cosh \beta_{0}(a-u)}{\epsilon_{0}\left(\beta_{0}^{2}-\nu^{2}\right)^{\frac{1}{2}}}\right], 0<y, u<h .
\end{gathered}
$$




\section{Case II}

$y, u \in(c, d)$

$$
\begin{gathered}
N^{S M M}(y, u)=\frac{\delta_{0} \mu}{\gamma_{0} \cosh ^{2} \gamma_{0} h}\left[\sum _ { n = 1 } ^ { \infty } \left(\frac{\gamma_{n} \cos \gamma_{n}(h-y) \cos \gamma_{n}(h-u)}{\delta_{n} s_{n}}+\right.\right. \\
\left.\left.\frac{\operatorname{coth} \zeta_{n} b}{2(d-c) \zeta_{n}} \cos \frac{n \pi(d-y)}{d-c} \cos \frac{n \pi(d-u)}{d-c}\right)+\frac{\operatorname{coth} \nu b}{4(d-c) \nu}\right] . \\
N^{A N S M M}(y, u)=\frac{\delta_{0} \mu}{\gamma_{0} \cosh ^{2} \gamma_{0} h}\left[\sum _ { n = 1 } ^ { \infty } \left(\frac{\gamma_{n} \cos \gamma_{n}(h-y) \cos \gamma_{n}(h-u)}{\delta_{n} s_{n}}+\right.\right. \\
\left.\left.\frac{\tanh \zeta_{n} b}{2(d-c) \zeta_{n}} \cos \frac{n \pi(d-y)}{d-c} \cos \frac{n \pi(d-u)}{d-c}\right)+\frac{\tanh \nu b}{4(d-c) \nu}\right] .
\end{gathered}
$$

\section{Case III}

$y \in(0, a), u \in(c, d)$ or $y \in(c, d), u \in(0, a)$

$$
N^{S M M, A N S M M}(y, u)=\frac{\delta_{0} \mu}{\gamma_{0} \cosh ^{2} \gamma_{0} h}\left[\sum_{n=1}^{\infty} \frac{\gamma_{n} \cos \gamma_{n}(h-y) \cos \gamma_{n}(h-u)}{\delta_{n} s_{n}}\right]
$$

Now if we define

$$
C^{S M M, A N S M M}=-i \frac{1-R^{S M M, A N S M M}}{1+R^{S M M, A N S M M}},
$$

then by using (29) and (39), we obtain

$$
C^{S M M, A N S M M}=\int_{(0, h)-L_{j}} F^{S M M, A N S M M}(y) \frac{\cosh \gamma_{0}(h-y)}{\cosh \gamma_{0} h} d y
$$

and $F^{S M M, A N S M M}(y)$ and $C^{S M M, A N S M M}$ are real quantities. Thus if the integral equations (39) can be solved, then these solutions can be used to evaluate $C^{S M M, A N S M M}$ from the relations (54), and these produce the actual reflection and transmission coefficients $|R|$ and $|T|$ by using

$$
|R|=\frac{\left|1+C^{S M M} C^{A N S M M}\right|}{\triangle} \text { and }|T|=\frac{\left|C^{S M M}-C^{A N S M M}\right|}{\triangle}
$$

with

$$
\triangle=\left\{1+\left(C^{S M M}\right)^{2}+\left(C^{A N S M M}\right)^{2}+\left(C^{S M M} C^{A N S M M}\right)^{2}\right\}^{\frac{1}{2}},
$$

which are obtained from equations (14) and (53).

\subsection{Solution of integral equation}

Utilizing Galerkin technique, we approximate $F^{S M M, A N S M M}(y)$ as

$$
F^{S M M, A N S M M}(y) \approx \mathcal{F}^{S M M, A N S M M}(y), y \in(0, h)-L_{j}, j=1,2,3
$$

$\mathcal{F}^{S M M, A N S M M}(y)$ is expanded in terms of multi-term Galerkin expansions with a suitable choice of basis function. In case of type-I barrier, there is a single interval but for type-II 
and type-III barriers there are two disjoint intervals.

For type-I,

$$
\mathcal{F}^{S M M, A N S M M}(y)=\sum_{n=0}^{N} a_{n}^{S M M, A N S M M} f_{n}^{S M M, A N S M M}(y), y \in(0, h)-L_{1}
$$

and for type-II

$$
\mathcal{F}^{S M M, A N S M M}(y)=\left\{\begin{array}{l}
\sum_{n=0}^{N} a_{n}^{S M M, A N S M M} p_{n}^{S M M, A N S M M}(y), 0<y<h \\
\sum_{n=0}^{N} b_{n}^{S M M, A N S M M} q_{n}^{S M M, A N S M M}(y), c<y<h
\end{array}\right.
$$

and for type-III

$$
\mathcal{F}^{S M M, A N S M M}(y)=\left\{\begin{array}{l}
\sum_{n=0}^{N} a_{n}^{S M M, A N S M M} p 1_{n}^{S M M, A N S M M}(y), 0<y<h \\
\sum_{n=0}^{N} b_{n}^{S M M, A N S M M} q 1_{n}^{S M M, A N S M M}(y), c<y<d
\end{array}\right.
$$

where the basis functions $f_{n}^{S M M, A N S M M}(y)$ for $a<y<h$ and $p_{n}^{S M M, A N S M M}(y)$ for $0<y<a, q_{n}^{S M M, A N S M M}(y)$ for $c<y<h$ and $p 1_{n}^{S M M, A N S M M}(y)$ for $0<y<a$, $q 1_{n}^{S M M, A N S M M}(y)$ for $c<y<d$ are taken in terms of ultraspherical Gegenbauer polynomials of order $1 / 6$ with suitable weights [cf. Porter[3]]. The basis functions in different intervals are given below.

\subsection{Basis functions}

\section{Type-I :}

For this type of fully submerged barrier, we have to take the surface tension condition and the behavior becomes

$$
F_{m}^{S M M, A N S M M} \sim(c-y)^{\frac{-1}{3}}
$$

as $y \rightarrow c^{-0}$ derived by considering the flow field near the corner point $(b, c)$. Thus here

$$
f_{m}^{S M M}(y)=f_{m}^{A N S M M}(y)=f_{m}(y)=-\frac{d}{d y}\left[e^{\frac{-K y}{\gamma+M \gamma^{3}}} \int_{y}^{c} e^{\frac{-K t}{\gamma+M \gamma^{3}}} \tilde{f}_{m}(t) d t\right], 0<y<c
$$

We choose the basis functions in terms of $\tilde{f}_{m}(y)$ as follows

$$
\tilde{f}_{m}(y)=\frac{2^{\frac{7}{6}} \Gamma\left(\frac{1}{6}\right)(2 m) !}{\pi \Gamma\left(2 m+\frac{1}{3}\right)(c)^{\frac{1}{3}}\left(c^{2}-y^{2}\right)^{\frac{1}{3}}} C_{2 m}^{\frac{1}{6}}\left(\frac{y}{c}\right), 0<y<c
$$

\section{Type II:}

Here we choose two different basis functions for two disjoint intervals.

$$
p_{m}^{S M M}(y)=p_{m}^{A N S M M}(y)=p_{m}(y)=-\frac{d}{d y}\left[e^{\frac{-K y}{\gamma+M \gamma^{3}}} \int_{y}^{a} e^{\frac{-K t}{\gamma+M \gamma^{3}}} \tilde{p}_{m}(t) d t\right], 0<y<a
$$


where

$$
\tilde{p}_{m}(y)=\frac{2^{\frac{7}{6}} \Gamma\left(\frac{1}{6}\right)(2 m) !}{\pi \Gamma\left(2 m+\frac{1}{3}\right)(a)^{\frac{1}{3}}\left(a^{2}-y^{2}\right)^{\frac{1}{3}}} C_{2 m}^{\frac{1}{6}}\left(\frac{y}{a}\right), 0<y<a
$$

and

$$
\begin{gathered}
q_{m}^{S M M}(y)=g 1_{m+1}^{(1)}(y), m=0,1,2, \ldots \\
q_{m}^{A N S M M}(y)=g 1_{m}^{(1)}(y), m=0,1,2, \ldots
\end{gathered}
$$

where

$$
g 1_{m}^{(1)}(y)=\frac{2^{\frac{7}{6}} \Gamma\left(\frac{1}{6}\right)(2 m) !}{\pi \Gamma\left(2 m+\frac{1}{3}\right)(h-c)^{\frac{1}{3}}\left((h-c)^{2}-(h-y)^{2}\right)^{\frac{1}{3}}} C_{2 m}^{\frac{1}{6}}\left(\frac{h-y}{h-c}\right), c<y<h
$$

\section{Type III:}

Here we choose two different basis functions for two disjoint intervals.

$$
p 1_{m}^{S M M}(y)=p 1_{m}^{A N S M M}(y)=p 1_{m}(y)=-\frac{d}{d y}\left[e^{\frac{-K y}{\gamma+M \gamma^{3}}} \int_{y}^{a} e^{\frac{-K t}{\gamma+M \gamma^{3}}} \tilde{x}_{m}(t) d t\right], 0<y<a
$$

where

$$
\tilde{p} 1_{m}(y)=\frac{2^{\frac{7}{6}} \Gamma\left(\frac{1}{6}\right)(2 m) !}{\pi \Gamma\left(2 m+\frac{1}{3}\right)(a)^{\frac{1}{3}}\left(a^{2}-y^{2}\right)^{\frac{1}{3}}} C_{2 m}^{\frac{1}{6}}\left(\frac{y}{a}\right), 0<y<a
$$

and

$$
q 1_{m}^{S M M, A N S M M}(y)=\frac{2^{\frac{7}{6}} \Gamma\left(\frac{1}{6}\right)(m) !}{\pi \Gamma\left(m+\frac{1}{3}\right)\left(\frac{d-c}{2}\right)^{\frac{1}{3}}((y-c)(d-y))^{\frac{1}{3}}} C_{m}^{\frac{1}{6}}\left(\frac{2 y-c-d}{d-c}\right), c<y<d
$$

As the horizontal velocity component near the corner points of the thick barriers of all the three types, have a cube root singularity, then allowing similar arguments as given in Kanoria et al. [10], here also the basis functions are chosen in terms of Gegenbauer polynomials of order $1 / 6$.

\subsection{Formation of linear system of equations}

For type I, we substitute the approximation (58) in equation (39), and then multiplying both side by appropriate $f^{S M M, A N S M M}(y)$ and integrate over $(a, h)$ to obtain the linear system of equations

$$
\sum_{n=0}^{N} a_{n}^{S M M, A N S M M} \chi_{m n}^{S M M, A N S M M}=\omega_{m}^{S M M, A N S M M}, \quad m=0,1, \ldots, N
$$


where

$$
\begin{gathered}
\chi_{m n}^{S M M, A N S M M}=\int_{a}^{h} \int_{a}^{h} N^{S M M, A N S M M}(y, u) f_{n}^{S M M, A N S M M}(u) f_{m}^{S M M, A N S M M}(y) d u d y \\
m, n=0,1,2, \ldots, N
\end{gathered}
$$

and

$$
\omega_{m}^{S M M, A N S M M}=\int_{a}^{h} \frac{\cosh \gamma_{0}(h-y)}{\cosh \gamma_{0} h} f_{m}^{S M M, A N S M M}(y) d y, m=0,1, \ldots, N .
$$

The integrals (71) and (72) can be evaluated explicitly, as in Kanoria et al. [10] by using the different properties and standard results on Gegenbaur polynomials. Thus the constants $a_{n}^{S M M, A N S M M}(n=0,1, \ldots N)$ are obtained by solving the linear equations (70). The relation (54) produce

$$
C^{S M M, A N S M M}=\sum_{n=0}^{N} a_{n}^{S M M, A N S M M} \omega_{n}^{S M M, A N S M M}
$$

so that $C^{S M M, A N S M M}$ are now found and $|R|$ and $|T|$ are evaluated from the relations (55).

For type II, we replace the equation (59) by (39) and multiplying both sides first by $p_{m}^{S M M, A N S M M}(y)(0<y<a)$ and then by $q_{m}^{S M M, A N S M M}(y)(c<y<h)$ and then integrate over $(0, a)$ and $(c, h)$ respectively, we get the linear system of equations as follows:

$$
\begin{gathered}
\sum_{n=0}^{N} a_{n}^{S M M, A N S M M}\left(\begin{array}{c}
\mathcal{G}_{m n}^{S M M, A N S M M} \\
\mathcal{P}_{m n}^{S M M, A N S M M}
\end{array}\right)+\sum_{n=0}^{N} b_{n}^{S M M, A N S M M}\left(\begin{array}{c}
\mathcal{H}_{m n}^{S M M, A N S M M} \\
\mathcal{Q}_{m n}^{S M M, A N S M M}
\end{array}\right) \\
=\left(\begin{array}{c}
\omega_{m}^{(1) S M M, A N S M M} \\
\omega_{m}^{(2) S M M, A N S M M}
\end{array}\right), m=0,1, \ldots, N
\end{gathered}
$$

where

$$
\begin{aligned}
\mathcal{G}_{m n}^{S M M, A N S M M} & =\int_{0}^{a}\left(\int_{0}^{a} N^{S M M, A N S M M}(y, u) p_{n}^{S M M, A N S M M}(u) d u\right) p_{m}^{S M M, A N S M M}(y) d y, \\
\mathcal{H}_{m n}^{S M M, A N S M M} & =\int_{0}^{a}\left(\int_{c}^{h} N^{S M M, A N S M M}(y, u) q_{n}^{S M M, A N S M M}(u) d u\right) p_{m}^{S M M, A N S M M}(y) d y, \\
\mathcal{P}_{m n}^{S M M, A N S M M} & =\int_{c}^{h}\left(\int_{0}^{a} N^{S M M, A N S M M}(y, u) p_{n}^{S M M, A N S M M}(u) d u\right) q_{m}^{S M M, A N S M M}(y) d y, \\
\mathcal{Q}_{m n}^{S M M, A N S M M} & =\int_{c}^{h}\left(\int_{c}^{h} N^{S M M, A N S M M}(y, u) q_{n}^{S M M, A N S M M}(u) d u\right) q_{m}^{S M M, A N S M M}(y) d y,
\end{aligned}
$$




$$
m, n=0,1,2, \ldots, N
$$

so that $\mathcal{P}_{m n}^{S M M, A N S M M}=\mathcal{H}_{m n}^{S M M, A N S M M}$, and

$$
\begin{gathered}
\omega_{m}^{(1) S M M, A N S M M}=\int_{0}^{a} \frac{\cosh \gamma_{0}(h-y)}{\cosh \gamma_{0} h} p_{m}^{S M M, A N S M M}(y) d y, \\
\omega_{m}^{(2) S M M, A N S M M}=\int_{c}^{h} \frac{\cosh \gamma_{0}(h-y)}{\cosh \gamma_{0} h} q_{m}^{S M M, A N S M M}(y) d y, \\
m=0,1, \ldots, N .
\end{gathered}
$$

The integrals in the relations (75) and (76) can be evaluated explicitly and thus the constants for type II barrier $a_{n}^{S M M, A N S M M}$ and $b_{n}^{S M M, A N S M M}(n=0,1,2, . . N)$ from linear equations (59) are obtained. We approximate $C^{S M M, A N S M M}$ as

$$
C^{S M M, A N S M M}=\sum_{n=0}^{N} a_{n}^{S M M, A N S M M} \omega_{n}^{(1) S M M, A N S M M}+\sum_{n=0}^{N} b_{n}^{S M M, A N S M M} \omega_{n}^{(2) S M M, A N S M M}
$$

For type III, we substitute the equation (60) in (39) and multiplying both sides first by $p 1_{m}^{S M M, A N S M M}(y)(0<y<a)$ and then by $q 1_{m}^{S M M, A N S M M}(y)(c<y<d)$ and then integrate over $(0, a)$ and $(c, d)$ respectively, we get the linear system of equations as follows:

$$
\begin{gathered}
\sum_{n=0}^{N} a_{n}^{S M M, A N S M M}\left(\begin{array}{c}
R_{m n}^{S M M, A N S M M} \\
X_{m n}^{S M M, A N S M M}
\end{array}\right)+\sum_{n=0}^{N} b_{n}^{S M M, A N S M M}\left(\begin{array}{c}
S_{m n}^{S M M, A N S M M} \\
Y_{m n}^{S M M, A N S M M}
\end{array}\right) \\
=\left(\begin{array}{c}
\omega 1_{m}^{(1) S M M, A N S M M} \\
\omega 1_{m}^{(2) S M M, A N S M M}
\end{array}\right), m=0,1, \ldots, N
\end{gathered}
$$

where

$$
\begin{aligned}
& R_{m n}^{S M M, A N S M M}=\int_{0}^{a}\left(\int_{0}^{a} N^{S M M, A N S M M}(y, u) p 1_{n}^{S M M, A N S M M}(u) d u\right) p 1_{m}^{S M M, A N S M M}(y) d y, \\
& S_{m n}^{S M M, A N S M M}=\int_{0}^{a}\left(\int_{c}^{d} N^{S M M, A N S M M}(y, u) q 1_{n}^{S M M, A N S M M}(u) d u\right) p 1_{m}^{S M M, A N S M M}(y) d y, \\
& X_{m n}^{S M M, A N S M M}=\int_{c}^{d}\left(\int_{0}^{a} N^{S M M, A N S M M}(y, u) p 1_{n}^{S M M, A N S M M}(u) d u\right) q 1_{m}^{S M M, A N S M M}(y) d y, \\
& Y_{m n}^{S M M, A N S M M}=\int_{c}^{d}\left(\int_{d}^{h} N^{S M M, A N S M M}(y, u) q 1_{n}^{S M M, A N S M M}(u) d u\right) q 1_{m}^{S M M, A N S M M}(y) d y,
\end{aligned}
$$




$$
m, n=0,1,2, \ldots, N,
$$

so that $X_{m n}^{S M M, A N S M M}=S_{m n}^{S M M, A N S M M}$, and

$$
\begin{gathered}
\omega 1_{m}^{(1) S M M, A N S M M}=\int_{0}^{a} \frac{\cosh \gamma_{0}(h-y)}{\cosh \gamma_{0} h} p 1_{m}^{S M M, A N S M M}(y) d y, \\
\omega 1_{m}^{(2) S M M, A N S M M}=\int_{c}^{d} \frac{\cosh \gamma_{0}(h-y)}{\cosh \gamma_{0} h} q 1_{m}^{S M M, A N S M M}(y) d y, \\
m=0,1, \ldots, N .
\end{gathered}
$$

Solving the integrals present in (79) and (80) explicitly, the constants for type III barrier $a_{n}^{S M M, A N S M M}$ and $b_{n}^{S M M, A N S M M}(n=0,1,2, . . N)$ from linear equations (60) are obtained. We evaluate $C^{S M M, A N S M M}$ as

$$
C^{S M M, A N S M M}=\sum_{n=0}^{N} a_{n}^{S M M, A N S M M} \omega 1_{n}^{(1) S M M, A N S M M}+\sum_{n=0}^{N} b_{n}^{S M M, A N S M M} \omega 1_{n}^{(2) S M M, A N S M M}
$$

\subsection{Coefficients of linear system of equations}

\section{Type I}

$$
\begin{aligned}
& \chi_{m n}^{S M M}=\frac{\delta_{0} \mu}{\gamma_{0} \cosh ^{2} \gamma_{0} h}\left[4 ( - 1 ) ^ { m + n } \sum _ { r = 1 } ^ { \infty } \left\{\frac{\gamma_{r} \cos ^{2} \gamma_{r} h}{\delta_{r} s_{r}\left(\gamma_{r} c\right)^{\frac{1}{3}}} J_{2 m+\frac{1}{6}}\left(\gamma_{r} c\right) J_{2 n+\frac{1}{6}}\left(\gamma_{r} c\right)\right.\right. \\
& \left.+\operatorname{coth} t_{r} b \frac{\alpha_{r} \cos ^{2} \alpha_{r} c}{\lambda_{r} t_{r}\left(\alpha_{r} c\right)^{\frac{1}{3}}} J_{2 m+\frac{1}{6}}\left(\alpha_{r} c\right) J_{2 n+\frac{1}{6}}\left(\alpha_{r} c\right)\right\} \\
& \left.+\left(-\cot \left(\alpha_{0}^{2}-\nu^{2}\right)^{\frac{1}{2}} b\right) \frac{\cosh ^{2} \alpha_{0} c}{\lambda_{0}\left(\alpha_{0}^{2}-\nu^{2}\right)^{\frac{1}{6}} c^{\frac{1}{3}}} I_{2 m+\frac{1}{6}}\left(\alpha_{0} c\right) I_{2 n+\frac{1}{6}}\left(\alpha_{0} c\right)\right] \\
& \chi_{m n}^{A N S M M}=\frac{\delta_{0} \mu}{\gamma_{0} \cosh ^{2} \gamma_{0} h}\left[4 ( - 1 ) ^ { m + n } \sum _ { r = 1 } ^ { \infty } \left\{\frac{\gamma_{r} \cos ^{2} \gamma_{r} h}{\delta_{r} s_{r}\left(\gamma_{r} c\right)^{\frac{1}{3}}} J_{2 m+\frac{1}{6}}\left(\gamma_{r} c\right) J_{2 n+\frac{1}{6}}\left(\gamma_{r} c\right)\right.\right. \\
& \left.+\tanh t_{r} b \frac{\alpha_{r} \cos ^{2} \alpha_{r} c}{\lambda_{r} t_{r}\left(\alpha_{r} c\right)^{\frac{1}{3}}} J_{2 m+\frac{1}{6}}\left(\alpha_{r} c\right) J_{2 n+\frac{1}{6}}\left(\alpha_{r} c\right)\right\} \\
& \left.+\left(\tan \left(\alpha_{0}^{2}-\nu^{2}\right)^{\frac{1}{2}} b\right) \frac{\cosh ^{2} \alpha_{0} c}{\lambda_{0}\left(\alpha_{0}^{2}-\nu^{2}\right)^{\frac{1}{6}} c^{\frac{1}{3}}} I_{2 m+\frac{1}{6}}\left(\alpha_{0} c\right) I_{2 n+\frac{1}{6}}\left(\alpha_{0} c\right)\right] \\
& \omega_{m}^{S M M, A N S M M}=\frac{I_{2 m+\frac{1}{6}}\left(\gamma_{0} c\right)}{\left(\gamma_{0} c\right)^{\frac{1}{6}}}, \quad m=0,1, \ldots, N .
\end{aligned}
$$

\section{Type II}




$$
\begin{aligned}
& \mathcal{G}_{m n}^{S M M}=\frac{\delta_{0} \mu}{\gamma_{0} \cosh ^{2} \gamma_{0} h}\left[4 ( - 1 ) ^ { m + n } \sum _ { r = 1 } ^ { \infty } \left\{\frac{\gamma_{r} \cos ^{2} \gamma_{r} h}{\delta_{r} s_{r}\left(\gamma_{r} a\right)^{\frac{1}{3}}} J_{2 m+\frac{1}{6}}\left(\gamma_{r} a\right) J_{2 n+\frac{1}{6}}\left(\gamma_{r} a\right)\right.\right. \\
& \left.+\operatorname{coth} u_{r} b \frac{\beta_{r} \cos ^{2} \beta_{r} a}{\epsilon_{r} u_{r}\left(\beta_{r} a\right)^{\frac{1}{3}}} J_{2 m+\frac{1}{6}}\left(\beta_{r} a\right) J_{2 n+\frac{1}{6}}\left(\beta_{r} a\right)\right\} \\
& \left.+\left(-\cot \left(\beta_{0}^{2}-\nu^{2}\right)^{\frac{1}{2}} b\right) \frac{\cosh ^{2} \beta_{0} a}{\epsilon_{0}\left(\beta_{0}^{2}-\nu^{2}\right)^{\frac{1}{6}} a^{\frac{1}{3}}} I_{2 m+\frac{1}{6}}\left(\beta_{0} a\right) I_{2 n+\frac{1}{6}}\left(\beta_{0} a\right)\right] \\
& \mathcal{G}_{m n}^{A N S M M}=\frac{\delta_{0} \mu}{\gamma_{0} \cosh ^{2} \gamma_{0} h}\left[4 ( - 1 ) ^ { m + n } \sum _ { r = 1 } ^ { \infty } \left\{\frac{\gamma_{r} \cos ^{2} \gamma_{r} h}{\delta_{r} s_{r}\left(\gamma_{r} a\right)^{\frac{1}{3}}} J_{2 m+\frac{1}{6}}\left(\gamma_{r} a\right) J_{2 n+\frac{1}{6}}\left(\gamma_{r} a\right)\right.\right. \\
& \left.+\tanh u_{r} b \frac{\beta_{r} \cos ^{2} \beta_{r} a}{\epsilon_{r} u_{r}\left(\beta_{r} a\right)^{\frac{1}{3}}} J_{2 m+\frac{1}{6}}\left(\beta_{r} a\right) J_{2 n+\frac{1}{6}}\left(\beta_{r} a\right)\right\} \\
& \left.+\left(\tan \left(\beta_{0}^{2}-\nu^{2}\right)^{\frac{1}{2}} b\right) \frac{\cosh ^{2} \beta_{0} a}{\epsilon_{0}\left(\beta_{0}^{2}-\nu^{2}\right)^{\frac{1}{6}} a^{\frac{1}{3}}} I_{2 m+\frac{1}{6}}\left(\beta_{0} a\right) I_{2 n+\frac{1}{6}}\left(\beta_{0} a\right)\right] \\
& \mathcal{H}_{m n}^{S M M}=4(-1)^{m+n+1} \frac{\delta_{0} \mu}{\gamma_{0} \cosh ^{2} \gamma_{0} h} \sum_{r=1}^{\infty} \frac{\gamma_{r} \cos \gamma_{r} h}{\delta_{r} s_{r}\left(\gamma_{r} a\right)^{\frac{1}{6}}} \\
& \frac{1}{\left(\gamma_{r}(h-c)\right)^{\frac{1}{6}}} J_{2 m+\frac{1}{6}}\left(\gamma_{r} a\right) J_{2 n+\frac{13}{6}}\left(\gamma_{r}(h-c)\right) \\
& \mathcal{H}_{m n}^{A N S M M}=\mathcal{H}_{m, n-1}^{S M M} \\
& \mathcal{P}_{m n}^{S M M}=\mathcal{H}_{m n}^{S M M} \\
& \mathcal{P}_{m n}^{A N S M M}=\mathcal{P}_{m-1, n}^{S M M} \\
& \mathcal{Q}_{m n}^{S M M}=\frac{\delta_{0} \mu}{\gamma_{0} \cosh ^{2} \gamma_{0} h}\left[( - 1 ) ^ { m + n } \sum _ { r = 1 } ^ { \infty } \left\{\frac{4 \gamma_{r}}{\delta_{r} s_{r}\left(\gamma_{r}(h-c)\right)^{\frac{1}{3}}}\right.\right. \\
& J_{2 m+\frac{13}{6}}\left(\gamma_{r}(h-c)\right) J_{2 n+\frac{13}{6}}\left(\gamma_{r}(h-c)\right) \\
& \left.\left.+\frac{2}{r \pi} \operatorname{coth} \frac{r \pi b}{h-c} \frac{J_{2 m+\frac{13}{6}}(r \pi) J_{2 n+\frac{13}{6}}(r \pi)}{(r \pi)^{\frac{1}{3}}}\right\}\right] \\
& \mathcal{Q}_{m n}^{A N S M M}=\frac{\delta_{0} \mu}{\gamma_{0} \cosh ^{2} \gamma_{0} h}\left[\frac{12 \pi b}{h-c} \frac{2^{\frac{1}{3}}}{\Gamma\left(\frac{1}{3}\right)^{4}} \delta_{0 n} \delta_{0 m}\right. \\
& +(-1)^{m+n} \sum_{r=1}^{\infty}\left\{\frac{4 \gamma_{r}}{\delta_{r} s_{r}\left(\gamma_{r}(h-c)\right)^{\frac{1}{3}}} J_{2 m+\frac{1}{6}}\left(\gamma_{r}(h-c)\right) J_{2 n+\frac{1}{6}}\left(\gamma_{r}(h-c)\right)\right.
\end{aligned}
$$




$$
\left.\left.+\frac{2}{r \pi} \tanh \frac{r \pi b}{h-c} \frac{J_{2 m+\frac{1}{6}}(r \pi) J_{2 n+\frac{1}{6}}(r \pi)}{(r \pi)^{\frac{1}{3}}}\right\}\right]
$$

where $\delta_{0 n}=1$ for $n=0$, and $\delta_{0 n}=0$ for $n \geq 1$ and $J^{\prime} s$ are Bessel functions of first kind and

$$
\begin{gathered}
\omega_{m}^{(1) S M M}=\frac{I_{2 m+\frac{1}{6}}\left(\gamma_{0} a\right)}{\left(\gamma_{0} a\right)^{\frac{1}{6}}}, \quad m=0,1, \ldots, N . \\
\omega_{m}^{(1) A N S M M}=\omega_{m}^{(1) S M M} \\
\omega_{m}^{(2) S M M}=\frac{1}{\cosh \gamma_{0} h} \frac{I_{2 m+\frac{7}{6}}\left(\gamma_{0}(h-c)\right)}{\left(\gamma_{0}(h-c)\right)^{\frac{1}{6}}}, m=0,1, \ldots, N . \\
\omega_{m}^{(2) A N S M M}=\omega_{m-1}^{(2) S M M}
\end{gathered}
$$

\section{Type III}

$$
\begin{aligned}
& R_{m n}^{S M M}=\frac{\delta_{0} \mu}{\gamma_{0} \cosh ^{2} \gamma_{0} h}\left[4 ( - 1 ) ^ { m + n } \sum _ { r = 1 } ^ { \infty } \left\{\frac{\gamma_{r} \cos ^{2} \gamma_{r} h}{\delta_{r} s_{r}\left(\gamma_{r} a\right)^{\frac{1}{3}}} J_{2 m+\frac{1}{6}}\left(\gamma_{r} a\right) J_{2 n+\frac{1}{6}}\left(\gamma_{r} a\right)\right.\right. \\
& \left.+\operatorname{coth} u_{r} b \frac{\beta_{r} \cos ^{2} \beta_{r} a}{\epsilon_{r} u_{r}\left(\beta_{r} a\right)^{\frac{1}{3}}} J_{2 m+\frac{1}{6}}\left(\beta_{r} a\right) J_{2 n+\frac{1}{6}}\left(\beta_{r} a\right)\right\} \\
& \left.+\left(-\cot \left(\beta_{0}^{2}-\nu^{2}\right)^{\frac{1}{2}} b\right) \frac{\cosh ^{2} \beta_{0} a}{\epsilon_{0}\left(\beta_{0}^{2}-\nu^{2}\right)^{\frac{1}{6}} a^{\frac{1}{3}}} I_{2 m+\frac{1}{6}}\left(\beta_{0} a\right) I_{2 n+\frac{1}{6}}\left(\beta_{0} a\right)\right] \\
& R_{m n}^{A N S M M}=\frac{\delta_{0} \mu}{\gamma_{0} \cosh ^{2} \gamma_{0} h}\left[4 ( - 1 ) ^ { m + n } \sum _ { r = 1 } ^ { \infty } \left\{\frac{\gamma_{r} \cos ^{2} \gamma_{r} h}{\delta_{r} s_{r}\left(\gamma_{r} a\right)^{\frac{1}{3}}} J_{2 m+\frac{1}{6}}\left(\gamma_{r} a\right) J_{2 n+\frac{1}{6}}\left(\gamma_{r} a\right)\right.\right. \\
& \left.+\tanh u_{r} b \frac{\beta_{r} \cos ^{2} \beta_{r} a}{\epsilon_{r} u_{r}\left(\beta_{r} a\right)^{\frac{1}{3}}} J_{2 m+\frac{1}{6}}\left(\beta_{r} a\right) J_{2 n+\frac{1}{6}}\left(\beta_{r} a\right)\right\} \\
& \left.+\left(\tan \left(\beta_{0}^{2}-\nu^{2}\right)^{\frac{1}{2}} b\right) \frac{\cosh ^{2} \beta_{0} a}{\epsilon_{0}\left(\beta_{0}^{2}-\nu^{2}\right)^{\frac{1}{6}} a^{\frac{1}{3}}} I_{2 m+\frac{1}{6}}\left(\beta_{0} a\right) I_{2 n+\frac{1}{6}}\left(\beta_{0} a\right)\right] \\
& S_{m n}^{S M M, A N S M M}=\frac{4 \delta_{0} \mu}{\gamma_{0} \cosh ^{2} \gamma_{0} h} \sum_{r=1}^{\infty} \frac{\gamma_{r} \cos \gamma_{r} h}{\delta_{r} s_{r}\left(\gamma_{r} a\right)^{\frac{1}{6}}} \\
& \frac{\cos \left(\frac{n \pi}{2}-\gamma_{r}\left(h-\frac{c+d}{2}\right)\right)}{\left(\gamma_{r} \frac{d-c}{2}\right)^{\frac{1}{6}}} J_{2 m+\frac{1}{6}}\left(\gamma_{r} a\right) J_{n+\frac{1}{6}}\left(\gamma_{r} \frac{d-c}{2}\right) \\
& S_{m n}^{S M M, A N S M M}=X_{m n}^{S M M, A N S M M} \\
& Y_{m n}^{S M M}=\frac{\delta_{0} \mu}{\gamma_{0} \cosh ^{2} \gamma_{0} h}\left[2 \sum _ { r = 1 } ^ { \infty } \left\{\frac{2 \gamma_{r}}{s_{r} \delta_{r}} \cos \left(\frac{n \pi}{2}-\gamma_{r}\left(h-\frac{c+d}{2}\right)\right)\right.\right.
\end{aligned}
$$




$$
\begin{gathered}
\cos \left(\frac{m \pi}{2}-\gamma_{r}\left(h-\frac{c+d}{2}\right)\right) \frac{J_{m+\frac{1}{6}}\left(\gamma_{r} \frac{d-c}{2}\right) J_{n+\frac{1}{6}}\left(\gamma_{r} \frac{d-c}{2}\right)}{\left(\gamma_{r} \frac{d-c}{2}\right)^{\frac{1}{3}}} \\
\left.+\frac{\operatorname{coth} \zeta_{r} b}{(d-c) \zeta_{r}} \cos \left\{\frac{m \pi}{2}-\frac{r \pi}{2}\right\} \cos \left\{\frac{n \pi}{2}-\frac{r \pi}{2}\right\} \frac{J_{m+1 / 6}\left(\frac{r \pi}{2}\right) J_{n+1 / 6}\left(\frac{r \pi}{2}\right)}{\left(\frac{r \pi}{2}\right)^{1 / 3}}\right\} \\
\left.+\frac{12 \pi}{(d-c)} \frac{2^{1 / 3}}{(\Gamma(1 / 3))^{4}} \frac{\operatorname{coth} \nu b}{\nu} \delta_{0 m} \delta_{0 n}\right]
\end{gathered}
$$

where $\delta_{0 n}=1$ for $n=0$, and $\delta_{0 n}=0$ for $n \geq 1$.

After replacing 'coth' by 'tanh', we obtain $Y_{m n}^{A N S M M}$ from (101).

$$
\begin{gathered}
\omega 1_{m}^{(1) S M M, A N S M M}=\frac{I_{2 m+1 / 6}\left(\gamma_{0} a\right)}{\left(\gamma_{0} a\right)^{\frac{1}{6}}} \\
\omega 1_{m}^{(2) S M M, A N S M M}=\frac{(-1)^{m} e^{\gamma_{0}\left(h-\frac{c+d}{2}\right)} e^{-\gamma_{0}\left(h-\frac{c+d}{2}\right)}}{2 \cosh \gamma_{0} h} \frac{I_{m+1 / 6}\left(\gamma_{0} \frac{d-c}{2}\right)}{\left(\gamma_{0} \frac{d-c}{2}\right)^{\frac{1}{6}}}
\end{gathered}
$$

where $I^{\prime} s$ are modified Bessel function of first kind.

\section{$4 \quad$ Numerical results and discussions}

The present numerical results describe the nature of reflection coefficient $|R|$ against the non dimensional wave numbers $K h$ for different values of the parameters $b / h, a / h$ and different values of incident angle $\theta$. For numerical computation of three type of barriers, we consider $N=10$ in equation (70), (74) and (78) respectively. The coefficients are obtained by employing $(N+1)$ Galerkin approximation technique. These are seen to satisfy the energy balance relations numerically. This gives a partial check on the correctness of the numerical result obtained here. We consider 200 terms in each series of $\chi_{m, n}^{S M M, A N S M M}$ and almost a six figure accuracy is obtained is obtained in the result. In the Table 1, we study the convergence of the analytical solution by showing the numerical values of $|R|$ for different values of $k h$ with the truncation size $N=2,5,7,10$ of the finite series (70) for type I barrier. From this table it is seen that the results for $|R|$ converge very rapidly with $N$. For $N=7$, an accuracy of almost five decimal places is achieved.

Table 1: Convergence of $|R|$ with $N$ for Type I Barrier with $b / h=0.5, c / h=0.5, \theta=$ $25^{0}, M / h^{2}=0.5$

\begin{tabular}{rrrrr}
\hline $\mathbf{K h}$ & $\mathbf{N}=2$ & $\mathbf{N}=5$ & $\mathbf{N}=7$ & $\mathbf{N}=10$ \\
\hline 0.2 & 0.35462 & 0.38562 & 0.395131 & 0.395133 \\
1.0 & 0.29652 & 0.31236 & 0.327913 & 0.327916 \\
1.8 & 0.13256 & 0.16235 & 0.175689 & 0.175692 \\
\hline
\end{tabular}

Table 2 shows the convergence of the numerical values of $|R|$ for different values of $k h$ with the truncation size $N=2,5,7,10$ of the finite series (74) for type II barrier. Here also a five figure accuracy is achieved for $N=7$. Thus, from these two tables, we can say 
the present method is quite efficient for the numerical calculation of $|R|$ (and $|T|)$. In a similar manner one can show the convergence analysis for Type III barrier. Here we avoid the similar calculations.

Table 2: Convergence of $|R|$ with $N$ for Type II Barrier with $a / h=0.4, b / h=1.0, c / h=$ $0.8, \theta=25^{0}, M / h^{2}=0.5$

\begin{tabular}{rrrrr}
\hline $\mathbf{K h}$ & $\mathbf{N}=2$ & $\mathbf{N}=5$ & $\mathbf{N}=7$ & $\mathbf{N}=10$ \\
\hline 0.2 & 0.31562 & 0.35841 & 0.361959 & 0.361966 \\
1.0 & 0.221024 & 0.261021 & 0.272900 & 0.272902 \\
1.8 & 0.361120 & 0.405543 & 0.417920 & 0.417921 \\
\hline
\end{tabular}

\subsubsection{Discussion of energy identity relation}

In Table 3, the numerical values of the components present in the energy identity relation are provided for different values of the dimensionless wavenumber $k h$ for Type I barrier with fixed values of $a / h=0.4, b / h=1.0, c / h=0.8, \theta=25^{0}, M / h^{2}=0.5$. It is observed from the table that the energy balance relation $|R|^{2}+|T|^{2}=1$ satisfied numerically. It shows a partial check of our present method. Thus numerically we can show that reflection and transmission coefficients are satisfied the energy balance relation, which is $|R|^{2}+|T|^{2}=1$. Thus in present numerical analysis, it is quite enough to show the behavior change of $|R|$ considering different parametric values.

Table 3: Energy identity, Type I barrier for $b / h=0.5, c / h=0.5, \theta=25^{0}, M / h^{2}=0.5$

\begin{tabular}{rrrr}
\hline Kh & $|R|$ & $|T|$ & $|R|^{2}+|T|^{2}$ \\
\hline 0.1 & 0.127612 & 0.991824 & 1.0000 \\
0.5 & 0.412045 & 0.911164 & 1.0000 \\
1.0 & 0.327916 & 0.944707 & 1.0000 \\
1.5 & 0.229463 & 0.969256 & 1.0000 \\
2.0 & 0.143046 & 0.989716 & 1.0000 \\
2.5 & 0.0729557 & 0.997335 & 1.0000 \\
2.9 & 0.0278562 & 0.999612 & 1.0000 \\
\hline
\end{tabular}

\subsubsection{Comparison with existing results}

In Fig. 3, the reflection coefficient verses non dimensional wave number for type I and type II barriers have been plotted without the effect of surface tension. For comparison with my present result with the figures given in Kanoria et. al's [10], we consider very small values of non dimension surface tension factor, $\frac{M}{h^{2}}=0.0001, \theta=0^{0}$ and other non dimensional parameters are taken same as Kanoria et. al's [10] data for their Figures 4 and 5. In Fig. 3, dashed line and thick line corresponds our present data line and star and thick dot points represent the data of Kanoria's Figure 4 and 5 respectively. We can say that our present figure 3 almost matched with those figures of Kanoria et al's[10] paper. This shows the partial check of the correctness of our present numerical data. 


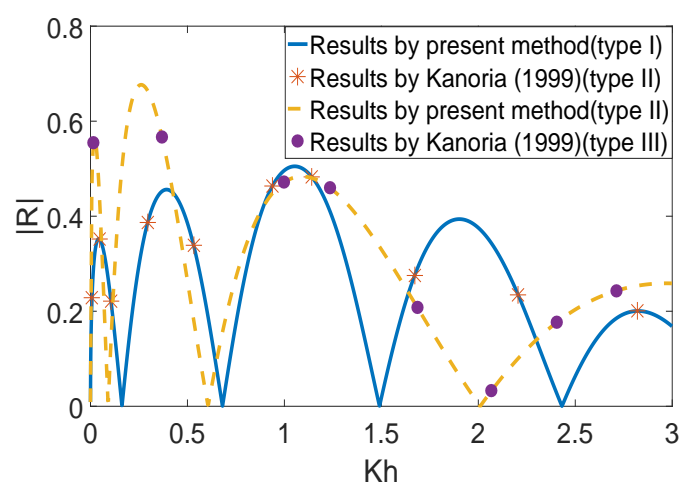

Figure 3: Reflection coefficients for two types of barriers without surface tension

\subsection{Effect of various parameters on reflection coefficients}

We now discuss the results associated with type I and type II barriers in separate figures. The variation of reflection coefficients $(|R|)$ as a function of wave numbers $(K h)$ are explained considering different parametric values.

\subsubsection{Type I barrier}

Figs. 4A, 4B and 5A, 5B show the plots of reflection coefficient $(|R|)$ of bottom standing thick barrier (Type I barrier) against non-dimensional wave number $(K h)$. Fig $4 \mathrm{~A}$ is drawn with fixed values of $\frac{c}{h}=0.5, \frac{M}{h^{2}}=1$ and $\theta=45^{0}$ for different values of width of the barrier $\frac{b}{h}=0.5,1.5$ and 2 . It is seen that increasing values of the width reflect less energy and also oscillation in the curve increases with increasing width of the barrier.

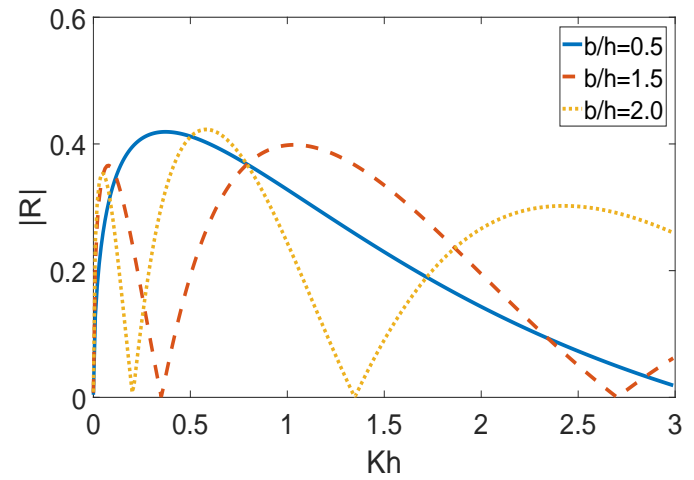

(A) $\frac{c}{h}=0.5, \frac{M}{h^{2}}=0.5, \theta=25^{0}$

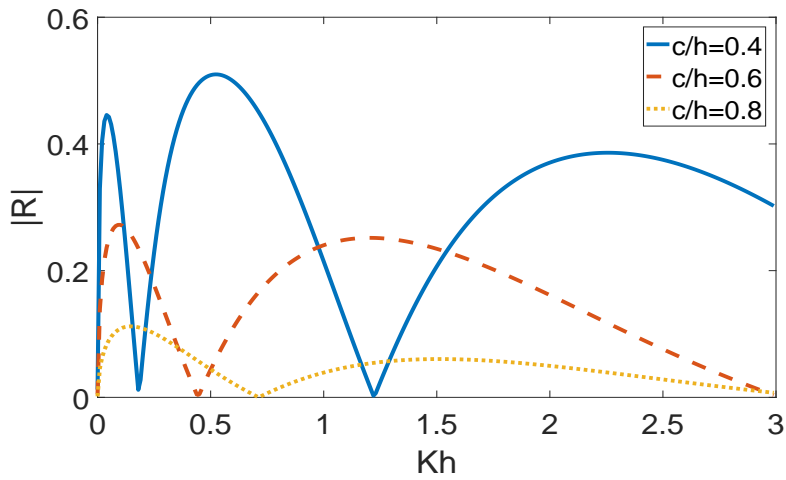

(B) $\frac{b}{h}=1.5, \frac{M}{h^{2}}=0.5, \theta=25^{0}$

Figure 4: Reflection coefficient for type I barrier (A)for different $\frac{b}{h}$; (B)for different $\frac{c}{h}$

In Fig. 4B, $|R|$ is depicted against $K h$ for different $\frac{c}{h}(=0.4,0.6,0.8)$ and consider fixed values of $\frac{b}{h}=2, \frac{M}{h^{2}}=0.5, \theta=25^{0}$. When depth from the free surface of the bottom standing thick barrier gradually increases less energy reflected and more energy transmitted. Zero reflection also decreases with increasing $\frac{c}{h}$. 


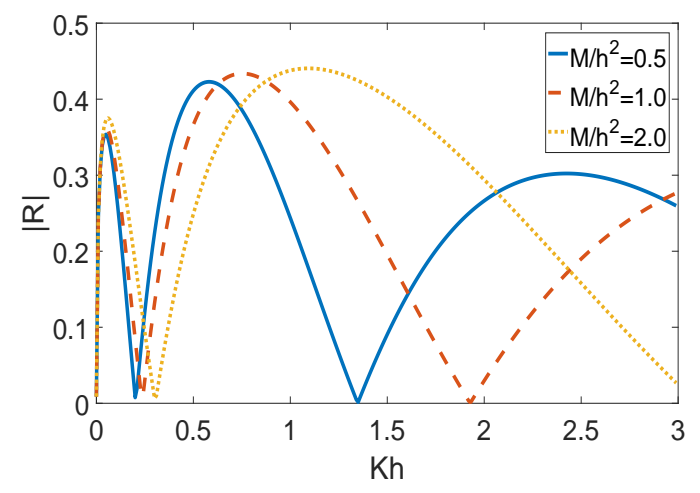

(A) $\frac{b}{h}=2, \frac{c}{h}=0.5, \theta=25^{0}$

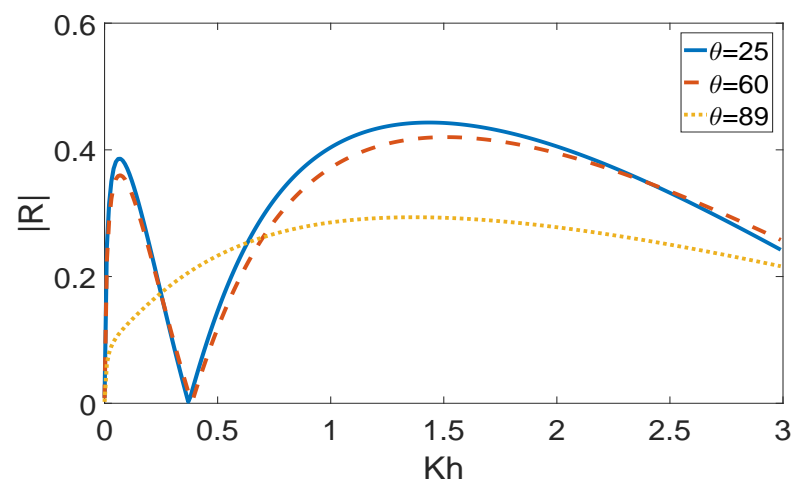

(B) $\frac{b}{h}=2, \frac{c}{h}=0.5, \frac{M}{h^{2}}=3$

Figure 5: Reflection coefficient for type I barrier (A)for different $\frac{M}{h^{2}}$; (B)for different $\theta$

The effect of surface tension on reflection coefficient is described in Fig. 5A which is drawn for three different values of surface tension factor $\frac{M}{h^{2}}=0.5,1.5$ and 2 with fixed values of $\frac{b}{h}=2, \frac{c}{h}=0.5, \theta=25^{0}$. It is clear that the amplitude of reflection coefficient decreases with increase of surface tension which agrees the presence of cohesive force in between the water molecule due to the surface tension. The graphs depicted in Fig. 5B are the reflection coefficients against wave numbers with fixed values of $\frac{b}{h}=2, \frac{c}{h}=0.5$ and $\frac{M}{h^{2}}=3$ and different values of incidence angle $\theta=25^{0}, 60^{\circ}, 89^{\circ}$. The peak values of $|R|$ gradually decreases with the increasing values of incidence wave angle. It is also noticed that when $\theta$ is almost $90^{\circ}$ the amplitude of $|R|$ is very small compared to the small incident angles.

\subsubsection{Type II barrier}

In Figs. 6A, 6B, 7A, 7B and 8, the variation of reflection energy coefficient is plotted against non dimensional wave number for type II barrier (submerged rectangular block). The graphs depicted in Fig. 6A are drawn with fixed values of $\frac{b}{h}=1, \frac{c}{h}=0.8, \frac{M}{h^{2}}=0.5$ and $\theta=$ $25^{0}$ and for different depth of the barrier from the upper surface of water, $\frac{a}{h}=0.4,0.5,0.6$. It is seen that increasing values of $\frac{a}{h}$ decreases the amplitude of reflection coefficient. Fig. $6 \mathrm{~B}$ shows the effect of change of width of the barrier on reflection coefficients by considering $\frac{b}{h}=1.0,0.7$ and 0.4 and fixed values of $\frac{a}{h}=0.5, \frac{c}{h}=0.8, \frac{M}{h^{2}}=0.5$ and $\theta=25^{0}$. Barrier with large width reflects more energy than the thinner one.

Fig. 7A is drawn for different values of $\frac{c}{h}(=0.5,0.7,0.9)$ and fixed values of $\frac{a}{h}=0.4, \frac{b}{h}=$ $1, \frac{M}{h^{2}}=0.5$ and $\theta=45^{\circ}$. From the figure it can be say that with increasing $\frac{c}{h},|R| \operatorname{grad}$ ually increases. It is physically quite obvious because large height of the barrier reflects more energy than the smaller one. Fig. $7 \mathrm{~B}$ is drawn for $|R|$ with different values of $\frac{M}{h^{2}}=0.5,1.0$, and1.5 and fixed values of $\frac{a}{h}=0.5, \frac{b}{h}=1, \frac{c}{h}=0.8$ and $\theta=25^{0}$. The effect of surface tension increases the amplitude of $|R|$ for type II barrier. Fig. 8 is plotted the variation of reflection coefficient $|R|$ against wave number $K h$ for different angles of incidence $\theta\left(=25^{0}, 50^{0}, 75^{0}\right.$ and $\left.89^{\circ}\right)$ and fixed values of $\frac{a}{h}=0.5, \frac{b}{h}=1, \frac{c}{h}=0.8$ and $\frac{M}{h^{2}}=0.5$. Figure shows that for presence of surface tension in the upper surface and submerged block 


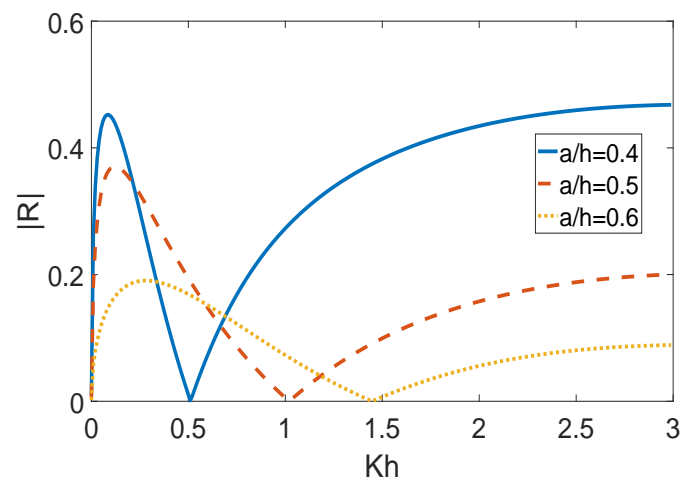

(A) $\frac{b}{h}=1, \frac{c}{h}=0.8, \frac{M}{h^{2}}=0.5, \theta=25^{0}$

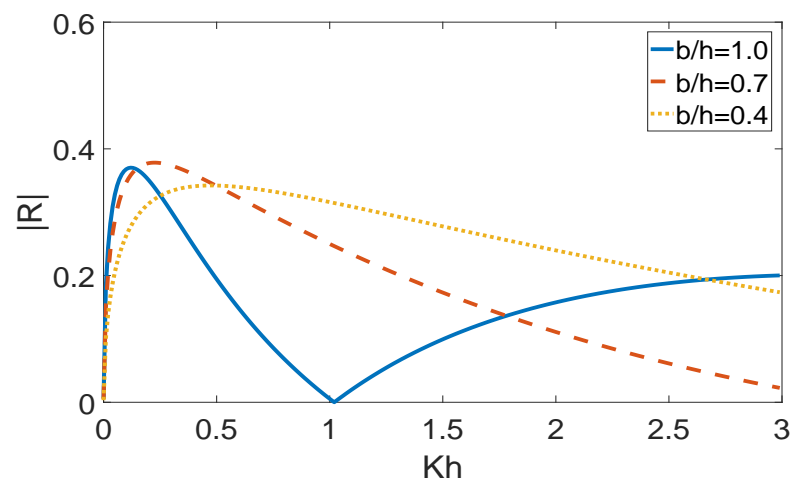

(B) $\frac{a}{h}=0.5, \frac{c}{h}=0.8, \frac{M}{h^{2}}=0.5, \theta=25^{0}$

Figure 6: Reflection coefficient for type II barrier (A)for different $\frac{a}{h}$; (B)for different $\frac{b}{h}$

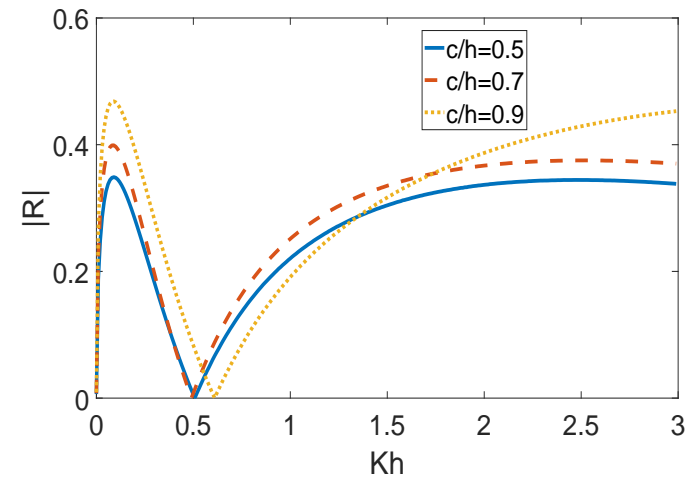

(A) $\frac{a}{h}=0.4, \frac{b}{h}=1, \frac{M}{h^{2}}=0.5, \theta=25^{0}$

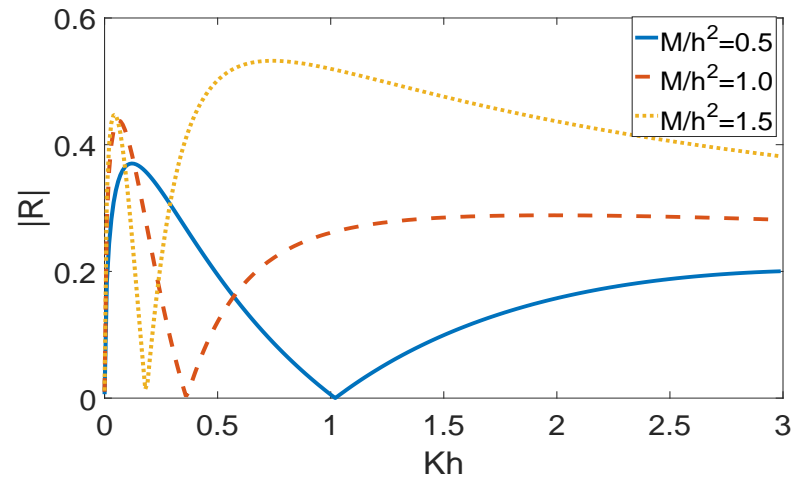

(B) $\frac{a}{h}=0.5, \frac{b}{h}=1, \frac{c}{h}=0.8, \theta=25^{0}$

Figure 7: Reflection coefficient for type II barrier (A)for different $\frac{c}{h}$; (B)for different $\frac{M}{h^{2}}$

in water, the amplitude of $|R|$ gradually increases with decreasing values of $\theta$.

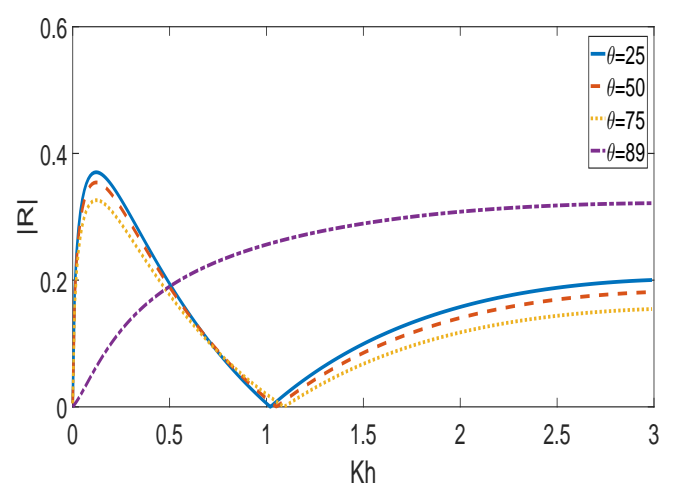

Figure 8: Reflection coefficient for type II barrier for different $\theta$ with $\frac{a}{h}=0.5, \frac{b}{h}=1, \frac{c}{h}=$ $0.8, \frac{M}{h^{2}}=0.5$

\subsubsection{Type III barrier}


Figs. 9A, 9B, 10 depict the behavior of $|R|$ against non dimensional wave numbers, for type III barrier. Fig. 9A is drawn for different values of width $\left(\frac{b}{h}=0.4,0.7\right.$ and 1.0$)$ of the thick barrier and fixed values of $\frac{a}{h}=0.2, \frac{M}{h^{2}}=0.5, \frac{(d-c)}{h}=0.3, \theta=25^{0}$. The figure have shown that increasing width of the barrier gradually increases the reflection coefficient and oscillating behavior in reflection curve also seen for large values of $\frac{b}{h}$.

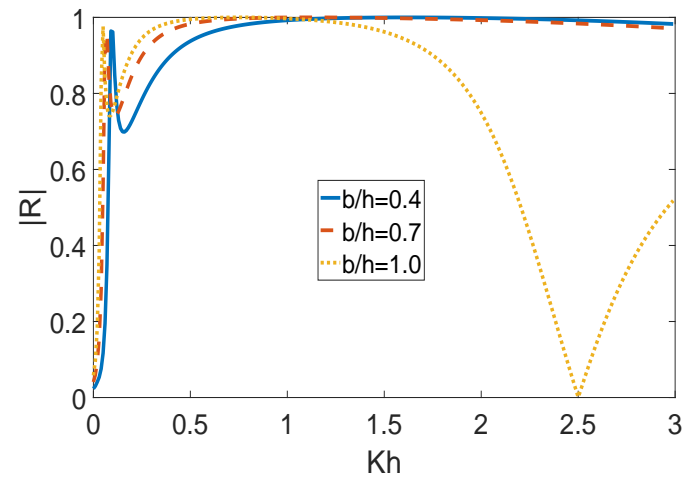

(A) $\frac{a}{h}=0.2, \frac{M}{h^{2}}=0.5, \frac{(d-c)}{h}=0.3, \theta=25^{0}$

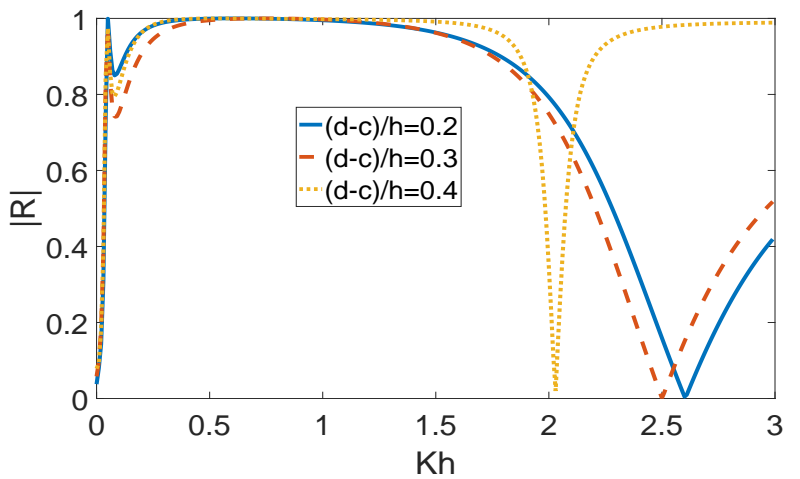

(B) $\frac{a}{h}=0.2, \frac{b}{h}=1, \frac{M}{h^{2}}=0.5, \theta=25^{0}$

Figure 9: Reflection coefficient for type III barrier (A)for different $\frac{b}{h}$; (B)for different $\frac{d-c}{h}$

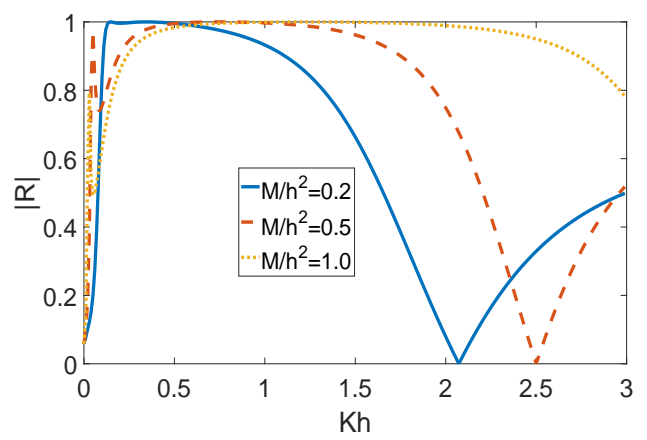

Figure 10: Reflection coefficient for type III barrier for different $\frac{M}{h}$ with $\frac{a}{h}=0.2, \frac{b}{h}=$ $1, \frac{(d-c)}{h}=0.3, \theta=25^{0}$.

In Fig. 9B, $|R|$ is depicted for various values of the gap of the barrier $\left(\frac{d-c}{h}=0.2,0.3\right.$ and 0.4$)$ and fixed values of $\frac{a}{h}=0.2, \frac{b}{h}=1, \frac{M}{h^{2}}=0.5, \theta=25^{0}$. The figure has clearly shown that amplitude and oscillation the reflection curve increases with large values of the gap in the barrier. Fig. 10 shows the behavior of $|R|$ for different values of $\frac{M}{h}=0.2,0.5$ and 1.0 with fixed values of $\frac{a}{h}=0.2, \frac{b}{h}=1, \frac{(d-c)}{h}=0.3, \theta=25^{0}$. Same as the type I and type II barriers, also in case of type III barrier, the effect of surface tension increases the amplitude of reflection coefficient of wave energy and it is more clear for large values of wave numbers. It can be noted that, all the reflection curves for each type of barriers with different parametric values show the zero reflection behavior. This phenomenon is true due to geometrical symmetry in the barrier's configuration. Also, number of zeros in reflection curve increases with increasing width of the barrier. For each type of barriers, the reflection curves for long waves confine near the origin. 


\section{Conclusion}

Oblique wave scattering by thick rectangular barrier in presence of surface tension at the upper surface have been studied here by employing multi-term Galerkin approximation technique. Three different geometrical positions of the barrier are considered here. The numerical estimate of reflection coefficient for different values of wave numbers and other parameters involved in the physical problem have been obtained and exemplify graphically. The numerical results also satisfy the energy balance relation. The results without surface tension have been recovered from present result by considering very small values of $M$. From the present study, it is clear that the amplitude of reflection coefficient decreases when the value of surface tension increases. The width and height of the thick barriers play a crucial role to the nature of scattering waves. The zero reflection and oscillation behavior of reflection coefficient are also observed here.

\section{Acknowledgment}

The authors would like to gratefully acknowledge the Editor and Reviewers for their valuable comments and suggestions to improve the quality of the manuscript.

\section{References}

[1] B.A. Packham, W.E. Williams, J. Inst. Maths Applics. 10 (1972) 176-184.

[2] I.J. Losada, M.A. Losada, A.J. Roldhn, Applied Ocean Research 14 (1992) 191-199.

[3] R. Porter, D,V. Evans, J Fluid Mech. 294 (1995) 155-180.

[4] B.N. Mandal, D.P. Dolai, Appl. Ocean Res. 16 (1994) 195-203.

[5] B.N. Mandal, M. Kanoria, J Offshore Mech Arctic Eng. 122 (2) (2000) 100-108.

[6] S. Banerjea, M. Kanoria, D. P. Dolai, B. N. Mandal, Appl Ocean Res. 18 (1996) 319-327.

[7] P. Das, D.P. Dolai, B.N. Mandal, J. Wtry. Port, Coastal and Ocean Engng. 123 (1997) 163-171.

[8] B.C. Das, S. De, B.N. Mandal, Journal of Engineering Mathematics 122 (2020) 81-99.

[9] C. Mei, J. Black, Journal of Fluid Mechanics 38 (3) (1969) 499-511.

[10] M. Kanoria, D. Dolai, B. Mandal, Journal of Engineering Mathematics 35 (1999) 361384 .

[11] B.N. Mandal, M. Kanoria, Proceedings of 17th International Conference on Offshore Mechanics and Arctic Engineering, ASME, Lisbon, Portugal, July(1998).

[12] D. Evans, In Proceedings of Cambridge Philosophical Society 64 (1968a) 833-847. 
[13] D. Evans, In Proceedings of Cambridge Philosophical Society 64 (1968b) 795-810.

[14] P. Rhodes-Robinson, Bulletin of the Australian Mathematical Society 2 (1970) 317-333.

[15] P. Rhodes-Robinson, In Proceedings of Cambridge Philosophical Society 70 (1971) 323337.

[16] P. Rhodes-Robinson, In Proceedings of Cam-bridge Philosophical Society 92 (1982) 369-373.

[17] A. Chakrabarti, T. Sahoo, Journal of the Australian Mathematical Society B 39 (1998) 539-556.

[18] L. Hocking, D. Mahdmina, Journal of Fluid Mechanics 224 (1991) 217-226.

[19] R. Chakraborty, B.N. Mandal, J Eng Math. 89 (2014) 101-112.

[20] R. Chakraborty, B.N. Mandal, ANZIAM J. 56 (2015) 286-298.

[21] J.N. Newman, J. Fluid Mech. 23 (1965) 23-29.

[22] A. Sasmal, S. Paul,S. Dey, J. Appl Fluid Mech. 12 (2019) 233-241.

[23] S. Paul, A. Sasmal, S. Dey, Ocean Engineering 193 (2019) 106613 (1-8).

[24] A. Sasmal, S. De, Journal of Ocean Engineering and Science 6 (2) (2021) 206-214.

[25] S. Paul, S. De, Ocean Engineering 220 (2021) 108449.

[26] D. Porter, Proc. Camb. Phil. Soc. 71 (1972) 411-421.

[27] D.V. Evans, M. Fernyhough, J. Fluid Mech. 297 (1995) 307-325. 